\title{
MANUFACTURING/REMANUFACTURING BASED SUPPLY CHAIN MANAGEMENT UNDER ADVERTISEMENTS AND CARBON EMISSIONS PROCESS
}

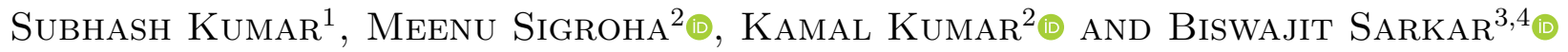

\begin{abstract}
One of the most successful ways to get the word out about a product's popularity across all types of customers is through advertising. It has a valuable direct influence on increasing product demand. The supply chain model is developed for manufacturer and retailer, where advertisements are dependent on demand. The advertisement rate has been considered a function that has enhanced at a diminishing rate concerning time, although the growth rate slowed. During the manufacturing cycle, the market's demand is a function of advertisement, and the customer's demand is a linear function of time. The production rate exceeds the demand rate during manufacturing and remanufacturing; shortages are not faced. It involves a manufacturing/remanufacturing process that quickly delivers consumer products and less waste. To keep the environment clean, the cost of carbon emissions is incorporated into the manufacturer's and supplier's holding and degrading costs. The model's primary purpose is to minimize the overall cost of manufacturing and remanufacturing. The overall cost during the manufacturing cycle is higher than that during the remanufacturing cycle. This study confirms that the increasing cost of advertising provides the continuous increasing value of the total cost. A numerical example is provided, graphical representation and sensitivity analysis determine the function's behavior and test the model.
\end{abstract}

Mathematics Subject Classification. 90B05, 90B06, 90B30, 90C30.

Received July 31, 2021. Accepted December 15, 2021.

\section{INTRODUCTION}

With the increasing competition in the global market, industrial players look into various methods to help them increase their sales and profits. Supply chain management (SCM) is a significant factor in any business process. It handles the production flow ranging from supply of raw material to delivery of final product out to

Keywords. Advertisements, carbon emissions, deterioration, remanufacturing, supply chain management.

1 Department of Mathematics, Meerut College, Meerut, Uttar Pradesh, India.

2 Department of Mathematics, Baba Mastnath University, Rohtak, India.

3 Department of Industrial Engineering, Yonsei University, 50 Yonsei-ro, Sinchon-dong, Seodaemun-gu, Seoul 03722, South Korea.

4 Center for Transdisciplinary Research (CFTR), Saveetha Dental College, Saveetha Institute of Medical and Technical Sciences, Saveetha University, 162, Poonamallee High Road, Velappanchavadi, Chennai, Tamil Nadu 600077, India.

*Corresponding author: bsbiswajitsarkar@gmail.com 
the customer. The main element of the supply chain are planning, source, production, delivery, and return. If the supply chain system is effective, it minimizes the production cycle's total cost, time, and wastage.

There is an increasing need to combat increasing carbon emissions and environmental degradation in today's era. The notion of manufacturing, recycling, and reverse logistics help achieve the goal. By remanufacturing, much of carbon is used again without being emitted as gases in the atmosphere. This process promotes the use of old or broken products resulting in the reduction of waste. The decline in carbon emissions leads us to a green environment. El Saadany et al. [13] analyzed an SCM model in which the product's carbon footprint determines demand. Habib et al. [16] established an optimization model that reduces the cost of overall biodiesel supply chain processes while reducing carbon emissions during operations.

Reverse logistics refers to the activities connected after the sale of a product to capture the value added and the life cycle of a product. It includes returning the product to the producer or recycling it. It is at a time called the aftermarket supply chain. During this change, the product has to go through various processes such as recycling, waste management, warehouse management, returns management, servicing, remanufacturing products being rebuilt with old or repaired parts, and refurbishment deals with the resale of repaired parts products. Ullah et al. [49] established a closed-loop SCM system for reverse logistics activities with stochastic demand and returns, which increases the cumulative uncertainty in the system to investigate optimal remanufacturing strategy.

Advertisement is done to publicity products, services, or events with the help of media platforms like newspapers, magazines, radios, television. It is designed so that it immediately attracts the attention of the people. It plays a crucial part in influencing the consumers' minds, tastes, and motives. It is essential in improving the sales of commodities or services. Business firms pay massive amounts to create an advertisement that helps in increasing the demand for their product in the market. It is a play of words with witty expressions and catchy punch lines that influence society. Manna et al. [25] analyzed a model for imperfect production in which advertising influences demand.

The sale of most items is dependent on its advertising in the public realm. As a result, advertising plays an essential role in enhancing a commodity's demand. Most of the existing literature considered SCM systems focused on carbon emissions and waste reduction manufacturing. This study considers advertising demand and carbon emissions under reverse logistics, according to the information of the authors, which has not been done in any research so far. Therefore, it fills the research gap that reverse logistics is not considered advertisement and carbon emissions costs. This study looks at advertisement demand, in which the rate of advertisement increases with time passing at a slower pace to record the sale before it is destroyed by deterioration. Demand depends on advertisement and time as demand largely impacts customers. An inventory model is developed with a single manufacturer and retailer by assuming that goods that have been remanufactured are as excellent as new items. Both manufacturing and remanufacturing models are separately formulated with various parameters and constant deterioration rates. Remanufacturing in world used products and reverse logistics. This study can be used in the textile industry, smart products, and other fields in the real world.

The following is a breakdown of the work: A literature review is in Section 2, the aim of the study, assumptions, and notations are defined in Section 3, and a mathematical model is formulated in Section 4; Section 5 explains the solution approach, numerical examples, and sensitivity are included in Sections 6 and 7. At last, Section 8 gives the conclusions and future research directions for the paper.

\section{LiterATURE REVIEW}

Over the years, researchers have agreed that inventory turns out to be a comprehensive study where inventory management is optimized. Today's problems related to economic production model (EPQ) include demand, deterioration of products, reverse logistics, and sustainability. Firstly, Harris developed the model with the assumption of constant demand. Silver and Meal [42] modified the model to include a variable demand rate. Donaldson [11], Lo et al. [24], and others developed a demand model under a linear trend. Time-dependent demand model was developed by Goyal and Giri [15], Silver and Peterson [43]. Karimi-Nasab et al. [19] discussed 
a price-dependent demand inventory model. Nowadays, advertisements are significant in controlling the demand for any product in the market. Cho [7] established a model with an optimal production and promotional policy for this purpose. Hazari et al. [17] examined a model for defective production with an advertisement policy in an uncertain environment. Khan et al. [20] created models for perishable items (with and without shortages), in which demand is driven by the frequency of advertising and the selling price of the commodity. Udayakumar et al. [50] studied a model for no instantaneously decaying items that consider money inflation and time discounting under advertisement-dependent demand, with the provider offering an acceptable wait period as an alternative to price discount. A smart SCM about variable lead time and variance under controllable production rate and advertise-dependent demand was proposed by Dey et al. [9].

By assuming an instantaneous repairable rate, Schardy [39] developed the rules for inventories with repairable products. The inventory model with limited storage capacity and finite repair rate was established by Nahmias and Rivera [26]. Conard [8] proposed a closed-loop supply chain (CLSC) model to examine the effect of customer issues on cost and market. Sebatjane and Adetunji [40] proposed a three-echelon supply chain (SC) model in which inventory levels and expiration dates determined the demand for increasing products. The policies for a CLSM model for green products with remanufacturing were developed by Chai et al. [5]. Inderfurth et al. [18] created a model for defective products subject to rework and deterioration. Widyadana and Wee [51] proposed a declining product manufacturing model that included a river and numerous production sets. Alamri [1] created a CLSC model for decaying products with dependent demand. Richter [30] created an economic ordered quantity (EOQ) model based on fixed demand and the condition that old products were repaired. El Saadany and Jaber [12] developed a CLSC model in which the returning rate is influenced by price and quality. Saxena et al. [37] determined a CLSC model with remanufacturing for the buyer/supplier. Rani et al. [27] established a CLSC model for decline items that took inflation and remanufacturing into account. Rani et al. [29] created a model that considered remanufacturing and learning effects. A CLSC model with remanufacturing was established by Liu et al. [23] and Aminipour et al. [2]. Sarkar et al. [36] produced a three-tiered sustainable SC model. Sarkar et al. [34] constructed a CLSC model to achieve circular economy by nullifying waste. According to Sarkar et al. [33], defective products might be reworked during each cycle when shortages were eliminated, and shortages could be reworked in the respective phase following the last cycle. Bhuniya et al. [4] described an energy-efficient smart production system in which production was variable and defective items were produced out of control. Preventive maintenance and restoration were utilized within the smart production system to avoid the out-ofcontrol state. Kugele et al. [35] proposed a smart production system that was depended on reliability of product. A geometric programming was used for finding optimal solutions. Bhuniya et al. [3] introduced a SCM model to avoid the backorder cost with constant and fuzzy demand. This model improves the quality of the products and reduces the vendor's setup cost by using the Kuhn-Tucker optimization technique.

Chang et al. [6] explored a production-inventory model with a multi-stage supply chain that uses preservation technology to prevent deterioration and boost investment. In the context of preservation technology investment, Kumar et al. [21] determined the optimal policies for deteriorating artifacts. Saha et al. [32] identified the optimum dynamic marketing investment. They proved the dynamic investment could control the market's demand. Even though the rate of deterioration is high, efficient preservation technology can be adopted to reduce the deterioration rate. Though the deterioration rate was reduced, wastes could be generated, which could not be zero by the preservation technology. The preservation technique only could reduce the rate of deterioration. Therefore, even though dynamic investment and preservation technology were used, the waste could not be fully controlled. This reduction of waste in a supply chain was initiated by Yadav et al. [48]. They introduced a new waste-free SC model. They proved that only preservation technology could reduce huge amounts of waste from product deterioration without having dynamic investment. Garai and Sarkar [38] investigated a waste reduction policy by remanufacturing utilizing through reverse logistics. Dey et al. [10] examined an autonomation policy in this model to find defective products from the production where the defective rate was random. Exponential demand was considered with safety stock and backorder. A multi-period multi-objective optimization problem of all three components of the business triad cost, quality, and time was managed by Tayyab and Sarkar [45] in 
TABLE 1. The author's previous research.

\begin{tabular}{|c|c|c|c|c|}
\hline Author (s) & Remanufacturing & $\begin{array}{l}\text { Type of } \\
\text { production }\end{array}$ & Demand & $\begin{array}{l}\text { Environmental } \\
\text { effect }\end{array}$ \\
\hline Sarkar et al. [34] & No & $\begin{array}{l}\text { Demand } \\
\text { dependent }\end{array}$ & Constant & $\begin{array}{l}\text { Costs effective under } \\
\text { circular economy }\end{array}$ \\
\hline Garai and Sarkar et al. [38] & Yes & No & Constant & $\begin{array}{l}\text { Waste } \\
\text { through } \\
\text { turing }\end{array}$ \\
\hline Rani et al. $[29]$ & Yes & Constant & $\begin{array}{l}\text { carbon } \\
\text { dependents }\end{array}$ & $\begin{array}{l}\text { Demand effective } \\
\text { under } \\
\text { carbon emissions }\end{array}$ \\
\hline Dey et al. [9] & No & $\begin{array}{l}\text { Flexible } \\
\text { production }\end{array}$ & $\begin{array}{l}\text { Advertisement } \\
\text { dependent }\end{array}$ & $\begin{array}{l}\text { Costs not effective } \\
\text { under } \\
\text { carbon emissions }\end{array}$ \\
\hline Saxena et al. [37] & Yes & Constant & Constant & $\begin{array}{l}\text { Costs not effective } \\
\text { under } \\
\text { carbon emissions }\end{array}$ \\
\hline Bhuniya et al. [4] & No & Constant & $\begin{array}{l}\text { Constant and } \\
\text { fuzzy } \\
\text { demands }\end{array}$ & $\begin{array}{l}\text { Costs not effective } \\
\text { under } \\
\text { carbon emissions }\end{array}$ \\
\hline Kumar et al. [22] & Yes & Linear & $\begin{array}{l}\text { Time- } \\
\text { dependent }\end{array}$ & $\begin{array}{l}\text { Demand effective } \\
\text { under } \\
\text { carbon emissions }\end{array}$ \\
\hline This study & Yes & Linear & $\begin{array}{l}\text { Advertisement } \\
\text { and time- } \\
\text { dependent }\end{array}$ & $\begin{array}{l}\text { Costs effective under } \\
\text { carbon emissions }\end{array}$ \\
\hline
\end{tabular}

a SCM model.

Kumar et al. [22] derived a CLSC model for smart objects using a reverse logistics system, with the retailer was taking carbon emissions into account. Ahmed et al. [28] presented reworking process using local storing facility and its effect in a global SCM. Sepehri et al. [41] created a model for degrading low-quality objects in which the pace of degradation was constant and might be controlled by investing in preservation technologies. Manufacturing processes emit carbon, which may be decreased by investing in tax. Gennady et al. [14] established a logistics and SC model in the face of uncertainty. For degrading products, Singh et al. [44] established a method that combined the effects of costs of carbon emissions and programs involving trade credits. Teng et al. [47] developed a system for remanufacturing under cap-and-trade legislation to minimize faulty items and limit carbon emissions, which was the manufacturer's primary goal in assuring sustainability. Sarkar et al. [49] developed a sustainable development framework for a cleaner textile production system with emissions tax and allocated cap policies for manufacturing. Table 1 shows some of the previous work.

\section{Assumptions \& notation}

The following aim, assumptions, and notation are used for this study.

\subsection{Aim of the study}

The focus of the study is on manufacturing/remanufacturing-based SCM in the context of advertisements and carbon emissions. During the manufacturing cycle, the market's demand is an advertisement-dependent function. To maintain the environment clean for both manufacturing and remanufacturing processes, carbon emissions costs have been added on deterioration costs and holding costs of items. This research is significant 


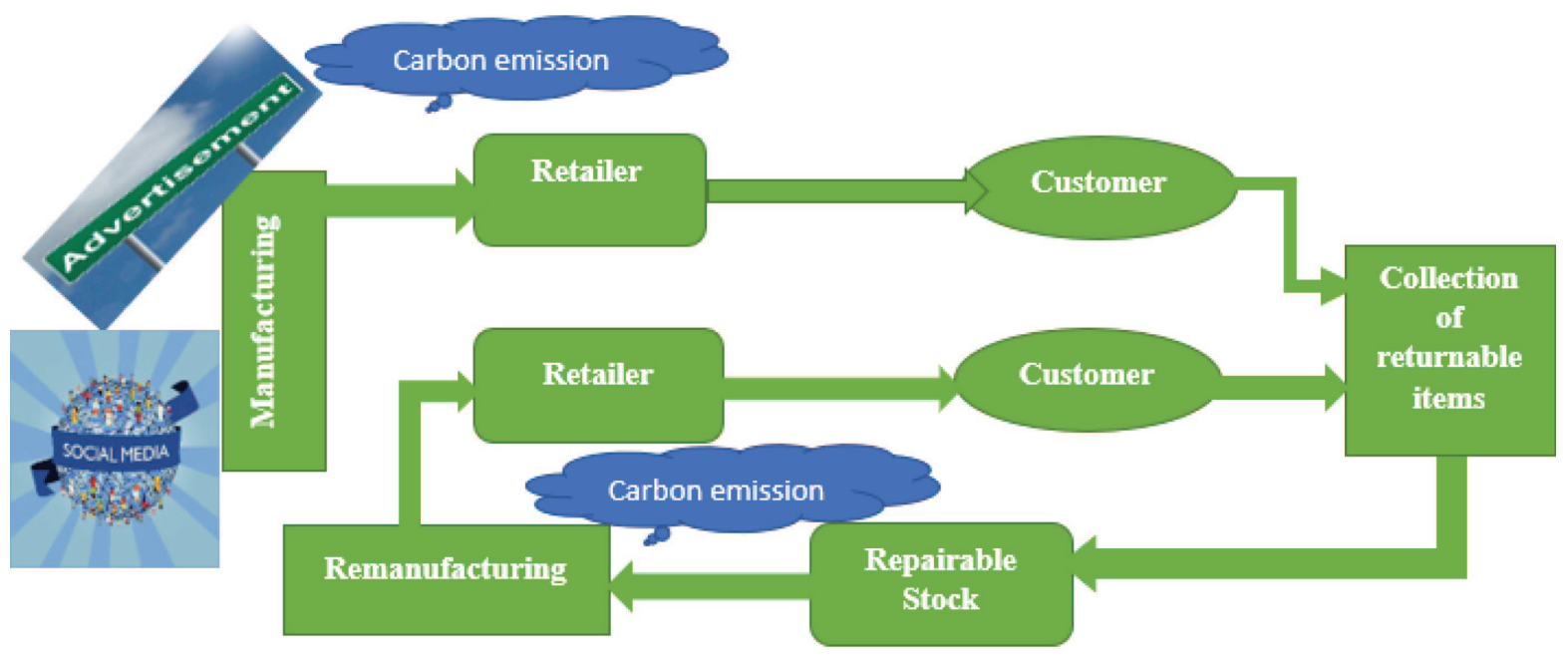

Figure 1. The flow of this study.

in advertising and remanufacturing procedures in the manufacturing industry. The study's flow is depicted in Figure 1.

\subsection{Assumptions}

(1) At this time, it is clear that the sale of an item is dependent on its advertising in the public sphere. As a result, advertising plays an essential role in enhancing a global commodity demand. In this way, we have looked at the demand function for ideal quality things affected by advertising. The demand rate function of advertising is

$$
D_{m}=D_{0} e^{-\lambda t}+\frac{a_{0}}{\lambda}\left(1-e^{-\lambda t}\right)+\frac{a_{1}}{a_{2}-\lambda}\left(e^{-a_{2} t}-e^{-\lambda t}\right) .
$$

As a result, the following differential equation describes the rate of change in demand for an item as $D_{m}^{\prime}=C_{\mathrm{ACM}}-\lambda D_{m}$ with $D(0)=D_{0}$. Where $a(t)=a_{0}-a_{1} e^{-a_{2} t}, 0<t<T_{t}$, where $a_{0}, a_{1} \& a_{2}$ are known parameters and $0<\lambda<1[25]$.

(2) This study considers carbon emissions and energy costs in terms of environmental criteria while calculating holding costs and deterioration costs for manufacturing and the manufacturing cycle [44].

(3) During manufacturing and remanufacturing, the production rate exceeds the rate of demand. In the beginning, the stock level is zero during manufacturing and remanufacturing [27].

(4) During manufacturing and remanufacturing, the production cycle is single. Lead time is zero [29].

(5) Remanufactured products have the exact cost and demand as they are like the new products [12,37].

(6) Returned products are gathered and put through a waste-recovery procedure. Parameter $\mu_{r}$ is the remanufactured product recovery rate [29].

(7) The rate of deterioration is different for manufacturing parameters and retailers' manufacturing parameters [22].

\subsection{Notation}

The model uses the following notation.

\section{Manufacturing parameters for supplier}

$D_{m} \quad$ Demand rate parameter: it is a function of advertisement and demand rate of market

$\alpha, \beta \quad$ Production rate parameters (units/unit time) for production $(\alpha+\beta t)$ where $\alpha>\beta$ 
$C_{\mathrm{SCM}} \quad$ Setup cost $(\$ /$ setup $)$

$C_{\mathrm{DCM}} \quad$ Deterioration cost $(\$ /$ unit)

$C_{\text {DCM1 }} \quad$ Carbon emissions cost owing to deterioration (\$/unit)

$C_{\mathrm{HCM}} \quad$ Holding cost (\$/unit/unit time)

$C_{\mathrm{HCM} 1}$ Costs for carbon emissions from holding items (\$/unit/unit time).

$C_{\text {PDCM }}$ Production cost (\$/unit)

$C_{\mathrm{PCM}} \quad$ Procurement cost

$C_{\mathrm{ACM}} \quad$ Advertisement cost (\$/advertisement)

$d_{1} \quad$ Deterioration rate

$a(t) \quad$ Advertisement rate

$\lambda \quad$ Depreciation rate

$Q_{m 1}(t) \quad$ At time $t$, inventory levels are at a certain level in the range $0 \leq t \leq t_{m 1}$

$Q_{m 2}(t)$ At time $t$, inventory levels are at a certain level in the range $t_{m 1} \leq t \leq t_{m 2}$

$I_{m} \quad$ Highest quantity during manufacturing

\section{Remanufacturing parameters for supplier}

$D_{r} \quad$ Demand rate parameter (units/unit time)

$\delta_{r} \quad$ Production rate parameter (units/unit time)

$C_{\mathrm{SCR}} \quad$ Setup cost $(\$ /$ setup $)$

$C_{\mathrm{DCR}} \quad$ Deterioration cost $(\$ /$ unit)

$C_{\mathrm{DCR} 1}$ Carbon emissions cost owing to deterioration (\$/unit).

$C_{\mathrm{HCR}} \quad$ Holding cost (\$/unit/unit time)

$C_{\mathrm{HCR} 1} \quad$ Costs include carbon emissions from holding items (\$/unit/unit time).

$C_{\mathrm{PDCR}} \quad$ Production cost $(\$ /$ unit)

$C_{\mathrm{PCR}} \quad$ Procurement cost $(\$ /$ unit)

$d_{1} \quad$ Deterioration rate

$t_{r 2} \quad$ Time when the inventory reaches the highest

$Q_{r 1}(t) \quad$ At time $t$, inventory levels are at a certain level in the range $t_{r 1} \leq t \leq t_{r 2}$

$Q_{r 2}(t) \quad$ At time $t$, inventory levels are at a certain level in the range $t_{r 1} \leq t \leq T_{t}$

$I_{r} \quad$ Highest quantity during manufacturing

\section{Collection parameters for collected products for retailer}

$I_{c} \quad$ Collected products level at $t=t_{m 2}$

$T_{c} \quad$ Time when the stock reaches zero

$Q_{c 1}(t) \quad$ At time $t$, inventory levels are at a certain level during the cycle of collection in the range $t_{r 1} \leq t \leq t_{c}$.

$Q_{c 2}(t) \quad$ At time $t$, inventory levels are at a certain level in the range $t_{c} \leq t \leq T_{c}$.

$\eta_{r} \quad$ Recovery rate

$\mu_{r} \quad$ Returned rate

\section{Retailer's parameters for manufacturing cycle}

$p, q \quad$ Demand rate parameter (units/unit time)

$\alpha, \beta \quad$ Production rate parameters (units/unit time) for production $(\alpha+\beta t)$ where $\alpha>\beta$.

$C_{\mathrm{PCRM}} \quad$ Purchasing cost $(\$ /$ unit)

$C_{\text {DCRM }} \quad$ Deterioration cost $(\$ /$ unit)

$C_{\text {DCRM1 }}$ Carbon emissions cost owing to deterioration ( $\$ /$ unit).

$C_{\mathrm{HCRM}}$ Holding cost $(\$ /$ unit/unit time)

$C_{\text {MCRM1 }}$ Carbon emissions cost $(\$ /$ unit/unit time)

$C_{\text {OCRM }}$ Ordering cost $(\$ /$ order $)$

$d_{2} \quad$ Deterioration rate

$t_{m} \quad$ Time when the inventory is zero 
$Q_{m}(t) \quad$ At time $t$, inventory levels are at a certain level in the range $0 \leq t \leq t_{m}$

$I_{r m} \quad$ Initial level of quantity during retailer's cycle

$C_{1} \quad$ Number of cycles

\section{Retailer's parameters for remanufacturing cycle}

$C_{\mathrm{PCRR}} \quad$ Purchasing cost (\$/unit)

$C_{\text {DCRR }} \quad$ Deterioration cost $(\$ /$ unit)

$C_{\text {DCRR1 }}$ carbon emissions cost owing to deterioration (\$/unit)

$C_{\mathrm{HCRR}} \quad$ Holding cost $(\$ /$ unit/unit time)

$C_{\mathrm{HCRR} 1}$ Costs include carbon emissions from holding items ( $\$ /$ unit/unit time).

$C_{\text {OCRR }} \quad$ Ordering cost $(\$ /$ order $)$

$t_{r} \quad$ Time when the inventory is zero

$Q_{r}(t) \quad$ At time $t$, inventory levels are at a certain level in the range $0 \leq t \leq t_{r}$

$C_{2} \quad$ Number of cycles

\section{Decision variables}

$t_{m 1} \quad$ Time when supplier's inventory is highest (time units)

$t_{m 2} \quad$ Time when supplier's inventory reaches a minimum (time units)

$t_{r 1} \quad$ Time when supplier's remanufacturing of inventory starts (time units)

$T_{t} \quad$ Time when supplier's inventory reaches the minimum (time units)

\section{Formulation of mathematical MODEL}

This model assumes that a single manufacturing cycle is followed by a single remanufacturing cycle. Manufacturing continues until $t_{m 1}$, at which point it is stopped. Stock is reduced due to demand and degradation. The overall cycle time is time $t_{m 2}$. Items that have been used are collected and returned to the supplier for recycling and remanufacturing. Production runs from $t_{m 2}$ to $t_{r 1}$ during remanufacturing, Afterwards, remanufacturing is halted. It is supposed that one production cycle has $C_{1}$ retail cycles and a single remanufacturing cycle has $C_{2}$ retail cycles, resulting in a total cycle time of $T_{t}$. The customer is thought to be concerned about the environment. The rate of deterioration is constant. Figure 2 shows this inventory flow.

\subsection{Manufacturing cycle of the supplier}

For Manufacturing cycle, level of inventory for suppliers at time $t$, rate of deterioration $d_{1}$, rate of production is linear $\alpha+\beta t$, and advertisement-dependent demand is $D_{m}$.

$$
\begin{aligned}
& \frac{\mathrm{d} Q_{m 1}(t)}{\mathrm{d} t}+d_{1} Q_{m 1}(t)=(\alpha+\beta t)-D_{m}, 0 \leq t \leq t_{m 1} \\
& \frac{\mathrm{d} Q_{m 2}(t)}{\mathrm{d} t}+d_{1} Q_{m 2}(t)=-D_{m}, t_{m 1} \leq t \leq t_{m 2} .
\end{aligned}
$$

From equation (4.1), one can find

$$
\begin{aligned}
& Q_{m 1}(t) e^{d_{1} t}=\int(\alpha+\beta t)-\left(D_{0} e^{-\lambda t}+\frac{a_{0}}{\lambda}\left(1-e^{-\lambda t}\right)+\frac{a_{1}}{a_{2}-\lambda}\left(e^{-a_{2} t}-e^{-\lambda t}\right)\right) e^{d_{1} t}+C_{m} \\
& Q_{m 1}(t) e^{d_{1} t}=\left[\begin{array}{l}
\frac{e^{d_{1} t}}{d_{1}}\left(\alpha-\frac{a_{0}}{\lambda}-\frac{\beta}{d_{1}}+\beta t\right)-\left(\frac{a_{1} e^{\left(d_{1}-a_{2}\right) t}}{\left(a_{2}-\lambda\right)\left(d_{1}-a_{2}\right)}\right) \\
-\left(\frac{e^{\left(d_{1}-\lambda\right) t}}{d_{1}-\lambda}\right)\left(D_{0}-\frac{a_{0}}{\lambda}-\frac{a_{1}}{a_{2}-\lambda}\right)
\end{array}\right]+C_{m}
\end{aligned}
$$

$$
\text { At } t=0, Q_{m 1}(t)=0
$$




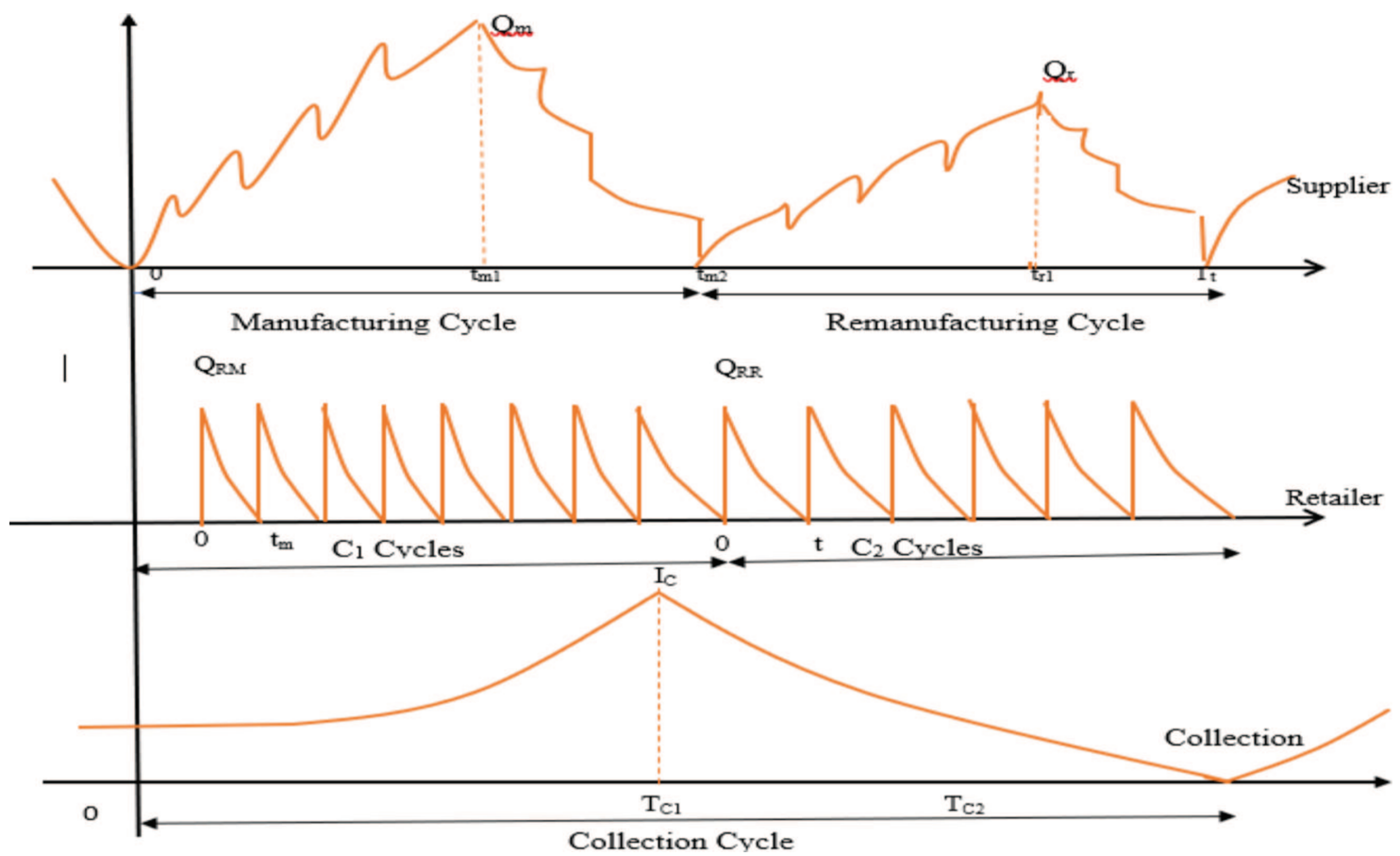

FiguRE 2. Inventory flow during supply chain.

$$
Q_{m 1}(t)=\left[\begin{array}{l}
\frac{\beta t}{d_{1}}+\frac{1}{d_{1}}\left\{\left(\alpha-\frac{a_{0}}{\lambda}-\frac{\beta}{d_{1}}\right)\left(1-e^{-d_{1} t}\right)\right\} \\
+\left(e^{-d_{1} t}-e^{-\lambda t}\right)\left(\frac{1}{d_{1}-\lambda}\right)\left(D_{0}-\frac{a_{0}}{\lambda}-\frac{a_{1}}{a_{2}-\lambda}\right) \\
+\left(e^{-d_{1} t}-e^{-a_{2} t}\right)\left(\frac{a_{1}}{\left(a_{2}-\lambda\right)\left(d_{1}-a_{2}\right)}\right)
\end{array}\right] .
$$

From equation (4.2), we get

$$
\begin{aligned}
& Q_{m 2}(t) e^{d_{1} t}=-\int\left(D_{0} e^{-\lambda t}+\frac{a_{0}}{\lambda}\left(1-e^{-\lambda t}\right)+\frac{a_{1}}{a_{2}-\lambda}\left(e^{-a_{2} t}-e^{-\lambda t}\right)\right) e^{d_{1} t} \mathrm{~d} t+C_{m 2} \\
& Q_{m 2}(t) e^{d_{1} t}=-\left[\begin{array}{l}
\left(\frac{e^{\left(d_{1}-\lambda\right) t}}{d_{1}-\lambda}\right)\left(D_{0}-\frac{a_{0}}{\lambda}-\frac{a_{1}}{a_{2}-\lambda}\right)+\frac{a_{0} e^{d_{1} t}}{\lambda d_{1}} \\
+\left(\frac{a_{1} e^{\left(d_{1}-a_{2}\right) t}}{\left(a_{2}-\lambda\right)\left(d_{1}-a_{2}\right)}\right)
\end{array}\right]+C_{m 2} \\
& \text { At } t=t_{m 2}, Q_{m 2}(t)=0 \\
& Q_{m 2}(t)=\left[\begin{array}{l}
\left(\frac{1}{d_{1}-\lambda}\right)\left(D_{0}-\frac{a_{0}}{\lambda}-\frac{a_{1}}{a_{2}-\lambda}\right)\left(e^{\left(d_{1}-\lambda\right) t_{m 2}}-e^{-\lambda t}\right)+\frac{a_{0}}{\lambda d_{1}}\left(e^{d_{1} t_{m 2}}-1\right) \\
+\left(\frac{a_{1}}{\left(a_{2}-\lambda\right)\left(d_{1}-a_{2}\right)}\right)\left(e^{\left(d_{1}-a_{2}\right) t_{m 2}}-e^{-a_{2} t}\right)
\end{array}\right] .
\end{aligned}
$$

Now, the following are various supplier costs.

\subsubsection{Setup cost}

This is a fixed cost for establishing the manufacturing substructure. It is calculated as follows:

$$
\mathrm{CM}_{\mathrm{SC}}=C_{\mathrm{SCM}} .
$$




\subsubsection{Advertisement cost}

The total cost of advertising in the manufacturing organization throughout the cycle is provided by

$$
\mathrm{CM}_{\mathrm{AC}}=C_{\mathrm{ACM}} \int_{0}^{t_{m 2}} a(t) \mathrm{d} t=C_{\mathrm{ACM}}\left[a_{0} t_{m 2}+\frac{a_{1}}{a_{2}}\left(e^{-a_{2} t_{m 2}}-1\right)\right] .
$$

\subsubsection{Production cost}

All expenditures incurred by a firm due to creating a product or offering a service are referred to as production costs. The following is a formula for calculating the rate of production.

$$
\mathrm{CM}_{\mathrm{PDC}}=C_{\mathrm{PDCM}} \int_{0}^{t_{m 1}}(\alpha+\beta t) \mathrm{d} t=C_{\mathrm{PDCM}}\left(\alpha t_{m 1}+\frac{\beta t_{m 1}^{2}}{2}\right) .
$$

\subsubsection{Procurement cost}

This includes a variety of expenses related to the acquisition of raw materials, equipment, and other goods during the production process and is calculated as follows:

$$
\begin{aligned}
& \mathrm{CM}_{\mathrm{PC}}=C_{\mathrm{PCM}} \int_{0}^{t_{m 1}} Q_{m 1}(t) \mathrm{d} t \\
& =C_{\mathrm{PCM}}\left[\begin{array}{l}
\frac{\beta t_{m 1}^{2}}{2 d_{1}}+\frac{1}{d_{1}^{2}}\left\{\left(\alpha-\frac{a_{0}}{\lambda}-\frac{\beta}{d_{1}}\right)\left(d_{1} t_{m 1}+e^{-d_{1} t_{m 1}}-1\right)\right\}+\left(1-e^{-d_{1} t_{m 1}}\right)\left(\frac{1}{d_{1}\left(d_{1}-\lambda\right)}\right) \\
\left(D_{0}-\frac{a_{0}}{\lambda}-\frac{a_{1}}{a_{2}-\lambda}\right)+\left(e^{-\lambda t_{m 1}}-1\right)\left(\frac{1}{\lambda\left(d_{1}-\lambda\right)}\right)\left(D_{0}-\frac{a_{0}}{\lambda}-\frac{a_{1}}{a_{2}-\lambda}\right) \\
+\left(1-e^{-d_{1} t_{m 1}}\right)\left(\frac{a_{1}}{d\left(a_{2}-\lambda\right)\left(d_{1}-a_{2}\right)}\right)+\left(e^{-a_{2} t_{m 1}}-1\right)\left(\frac{a_{1}}{a_{2}\left(a_{2}-\lambda\right)\left(d_{1}-a_{2}\right)}\right)
\end{array}\right] .
\end{aligned}
$$

\subsubsection{Deterioration cost}

The cost of deterioration includes the costs of spoilage, wear and tear, expiration, and other variables that cause the value of the stock in hand to decrease.

In this model, deteriorating cost, which includes together the conventional deteriorating cost $\left(C_{\mathrm{DCM}}\right)$ and the carbon emissions cost $\left(C_{\mathrm{DCM} 1}\right)$ caused by decaying products, are given by

$$
\begin{aligned}
& \mathrm{CM}_{\mathrm{DC}}=\left(C_{\mathrm{DCM}}+C_{\mathrm{DCM} 1}\right)\left[\int_{0}^{t_{m 1}} d_{1} Q_{m 1}(t) \mathrm{d} t+\int_{t_{m 1}}^{t_{m 2}} d_{1} Q_{m 2}(t) \mathrm{d} t\right] \\
& =\left(C_{\mathrm{PCM}}+C_{\mathrm{DCM} 1}\right) d_{1}\left[\begin{array}{l}
\frac{\beta t_{m 1}^{2}}{2 d_{1}}+\frac{1}{d_{1}^{2}}\left\{\left(\alpha-\frac{a_{0}}{\lambda}-\frac{\beta}{d_{1}}\right)\left(d_{1} t_{m 1}+e^{-d_{1} t_{m 1}}\right)\right\} \\
+\left(\frac{1}{\left(d_{1}-\lambda\right)}\right)\left(D_{0}-\frac{a_{0}}{\lambda}-\frac{a_{1}}{a_{2}-\lambda}\right)\left(\frac{1}{d_{1}}\left(1-e^{-d_{1} t_{m 1}}\right)\right. \\
\left.+\left(t_{m 2}-t_{m 1}\right) e^{\left(d_{1}-\lambda\right) t_{m 2}}\right) \\
+\left(e^{-\lambda t_{m 2}}-1\right)\left(\frac{1}{\lambda\left(d_{1}-\lambda\right)}\right)\left(D_{0}-\frac{a_{0}}{\lambda}-\frac{a_{1}}{a_{2}-\lambda}\right) \\
+\left(e^{-a_{2} t_{m 1}}-1\right)\left(\frac{a_{1}}{a_{2}\left(a_{2}-\lambda\right)\left(d_{1}-a_{2}\right)}\right) \\
+\left(\frac{a_{1}}{d\left(a_{2}-\lambda\right)\left(d_{1}-a_{2}\right)}\right)\left(\frac{1}{d_{1}}\left(1-e^{-d_{1} t_{m 1}}\right)+\left(t_{m 2}-t_{m 1}\right) e^{\left(d_{1}-a_{2}\right) t_{m 2}}\right) \\
+\frac{a_{0}}{\lambda d_{1}}\left(t_{m 2}-t_{m 1}\right)\left(e^{d_{1} t_{m 2}}-1\right)
\end{array}\right] .
\end{aligned}
$$

\subsubsection{Holding cost}

Rent, security, storage space, and insurance are just a few of the expenditures associated with storing inventory.

There are two components to the holding cost in this case. One component is related with product holding as $C_{\mathrm{HCM}}$, while the other is carbon emissions as $C_{\mathrm{HCM} 1}$. Consequently, the total cost of holding is

$$
\mathrm{CM}_{\mathrm{HCM}}=\left(C_{\mathrm{HCM}}+C_{\mathrm{HCM} 1}\right)\left[\int_{0}^{t_{m 1}} Q_{m 1}(t) \mathrm{d} t+\int_{t_{m 1}}^{t_{m 2}} Q_{m 2}(t) \mathrm{d} t\right]
$$




$$
=\left(C_{\mathrm{HCM}}+C_{\mathrm{HCM} 1}\right)\left[\begin{array}{l}
\frac{\beta t_{m 1}^{2}}{2 d_{1}}+\frac{1}{d_{1}^{2}}\left\{\left(\alpha-\frac{a_{0}}{\lambda}-\frac{\beta}{d_{1}}\right)\left(d_{1} t_{m 1}+e^{-d_{1} t_{m 1}}\right)\right\} \\
+\left(\frac{1}{\left(d_{1}-\lambda\right)}\right)\left(D_{0}-\frac{a_{0}}{\lambda}-\frac{a_{1}}{a_{2}-\lambda}\right)\left(\frac{1}{d_{1}}\left(1-e^{-d_{1} t_{m 1}}\right)\right. \\
\left.+\left(t_{m 2}-t_{m 1}\right) e^{\left(d_{1}-\lambda\right) t_{m 2}}\right) \\
+\left(e^{-\lambda t_{m 2}}-1\right)\left(\frac{1}{\lambda\left(d_{1}-\lambda\right)}\right)\left(D_{0}-\frac{a_{0}}{\lambda}-\frac{a_{1}}{a_{2}-\lambda}\right) \\
+\left(e^{-a_{2} t_{m 1}}-1\right)\left(\frac{a_{1}}{a_{2}\left(a_{2}-\lambda\right)\left(d_{1}-a_{2}\right)}\right) \\
+\left(\frac{a_{1}}{d\left(a_{2}-\lambda\right)\left(d_{1}-a_{2}\right)}\right)\left(\frac{1}{d_{1}}\left(1-e^{-d_{1} t_{m 1}}\right)+\left(t_{m 2}-t_{m 1}\right) e^{\left(d_{1}-a_{2}\right) t_{m 2}}\right) \\
+\frac{a_{0}}{\lambda d_{1}}\left(t_{m 2}-t_{m 1}\right)\left(e^{d_{1} t_{m 2}}-1\right)
\end{array}\right] .
$$

The total average cost for a supplier is calculated as

$$
(\mathrm{TCM})_{M}=\frac{1}{t_{m 2}}\left(\mathrm{CM}_{\mathrm{SC}}+\mathrm{CM}_{\mathrm{AC}}+\mathrm{CM}_{\mathrm{PDC}}+\mathrm{CM}_{\mathrm{PC}}+\mathrm{CM}_{\mathrm{DC}}+\mathrm{CM}_{\mathrm{HCM}}\right) .
$$

\subsection{Retailer's inventory from manufacturing cycle}

By assuming $d_{1}$ as the rate of deterioration and demand as a linear function, the level of inventory at time $t$ is given as

$$
\begin{aligned}
\frac{\mathrm{d} Q_{m}(t)}{\mathrm{d} t}+d_{2} Q_{m}(t) & =-(p+q t), 0 \leq t \leq t_{m} \\
\text { At } t & =t_{m}, Q_{m}(t)=0 \\
Q_{m}(t) e^{d_{2} t} & =-\int(p+q t) e^{d_{2} t} \mathrm{~d} t \\
Q_{m}(t) & =\left[\frac{e^{d_{2}\left(t_{m}-t\right)}}{d_{2}}\left(p+q t_{m}-\frac{q}{d_{2}}\right)-\frac{1}{d_{2}}\left(p+q t-\frac{q}{d_{2}}\right)\right] .
\end{aligned}
$$

The following are the different retailer costs.

Ordering cost $\mathrm{CR}_{\mathrm{OCR}}=C_{\mathrm{OCRM}}$.

Purchasing cost $\mathrm{CR}_{\mathrm{PCR}}=C_{\mathrm{PCRM}} Q_{m}(t=0)$

$$
=C_{\mathrm{PCRM}}\left[\frac{e^{d_{2} t_{m}}}{d_{2}}\left(p+q t_{m}-\frac{q}{d_{2}}\right)-\frac{1}{d_{2}}\left(p-\frac{q}{d_{2}}\right)\right] .
$$

Deterioration cost $\mathrm{CR}_{\mathrm{DCR}}=\left(C_{\mathrm{DCRM}}+C_{\mathrm{DCRM} 1}\right) \int_{0}^{t_{m}} d_{2} Q_{m}(t) \mathrm{d} t$

$$
=\left(C_{\mathrm{DCRM}}+C_{\mathrm{DCRM} 1}\right)\left[\frac{1}{d_{2}}\left(p+q t_{m}-\frac{q}{d_{2}}\right)\left(e^{d_{2} t_{m}}-1\right)-t_{m}\left(p+q t_{m}-\frac{q}{d_{2}}\right)\right] .
$$

Holding cost $\mathrm{CR}_{\mathrm{HCR}}=\left(C_{\mathrm{HCRM}}+C_{\mathrm{HCRM} 1}\right) \int_{0}^{t_{m}} Q_{m}(t) \mathrm{d} t$

$$
=\left(C_{\mathrm{HCRM}}+C_{\mathrm{HCRM}}\right)\left(\frac{1}{d_{2}}\right)\left[\frac{1}{d_{2}}\left(p+q t_{m}-\frac{q}{d_{2}}\right)\left(e^{d_{2} t_{m}}-1\right)-t_{m}\left(p+q t_{m}-\frac{q}{d_{2}}\right)\right] .
$$

For retailer's model, the total average cost is calculated as

$$
(\mathrm{TCR})_{M}=\frac{1}{t_{m}}\left(\mathrm{CR}_{\mathrm{OCR}}+\mathrm{CR}_{\mathrm{PCR}}+\mathrm{CR}_{\mathrm{DCR}}+\mathrm{CR}_{\mathrm{HCR}}\right) .
$$

This study assumes that total cycles for retailer manufactured products are $C_{1}$. Cycle time for the retailer is $t_{m}$. So, $t_{m}$ is written as

$$
t_{m}=\frac{t_{m 2}}{C_{1}} .
$$


As a consequence, the overall average cost of the manufacturing cycle is

$$
\begin{aligned}
& \text { Total cost }\left[\mathrm{TC}_{M}\left(t_{m 1}, t_{m 2}\right)\right]=(\mathrm{TCM})_{M}+(\mathrm{TCR})_{M} \\
& \mathrm{TC}_{M}\left(t_{m 1}, t_{m 2}\right)=\frac{1}{t_{m 2}}\left(\mathrm{CM}_{\mathrm{SC}}+\mathrm{CM}_{\mathrm{AC}}+\mathrm{CM}_{\mathrm{PDC}}+\mathrm{CM}_{\mathrm{PC}}+\mathrm{CM}_{\mathrm{DC}}+\mathrm{CM}_{\mathrm{HCM}}\right) \\
& +\frac{C_{1}}{t_{m 2}}\left(\mathrm{CR}_{\mathrm{OCR}}+\mathrm{CR}_{\mathrm{PCR}}+\mathrm{CR}_{\mathrm{DCR}}+\mathrm{CR}_{\mathrm{HCR}}\right) .
\end{aligned}
$$

\subsection{Collection inventory}

Consumers return used products to retailers, transporting them back to the supplier. The return rate for all products is same. After that, the products are treated and remanufactured. During remanufacturing, assuming $\eta_{r}, \mu_{r}$ and $\delta_{r}$ as the returned rate, recovery rate, and production rate parameter. The level of collection inventory is determined by

$$
\begin{aligned}
\frac{\mathrm{d} Q_{C 1}(t)}{\mathrm{d} t} & =\eta_{r} \mu_{r}\left(D_{r}+D_{0}\right)-\delta_{r}, T_{C 1} \leq t \leq T_{C 2} \\
\text { At } t & =T_{C 1}, Q_{C 1}(t)=I_{C} \\
Q_{C 1}(t) & =\left[\eta_{r} \mu_{r}\left(D_{r}+D_{0}\right)-\delta_{r}\right]\left(t-I_{C 1}\right)+I_{C} \\
\frac{\mathrm{d} Q_{C 2}(t)}{\mathrm{d} t} & =\eta_{r} \mu_{r}\left(D_{r}+D_{0}\right), T_{C 2} \leq t \leq T_{t+t_{c 1}} \\
\text { At } t & =T_{C 2}, Q_{C 1}(t)=0, Q_{C 2}(t)=0 \\
Q_{C 2}(t) & =\left[\eta_{r} \mu_{r}\left(D_{r}+D_{0}\right)\right]\left(t-T_{C 2}\right) \\
\text { At } t & =T_{t}+T_{C 2}, Q_{C 2}(t)=I_{C} \\
\delta_{r} & =\left[\eta_{r} \mu_{r}\left(D_{r}+D_{0}\right)\right]-\frac{I_{C}}{T_{C 1}-T_{C 2}} \\
T_{C 1}-T_{C 2} & =T_{t}-\frac{I_{C}}{\eta_{r} \mu_{r}\left(D_{r}+D_{0}\right)} \cdot
\end{aligned}
$$

\subsection{Remanufacturing cycle of the supplier}

Using the production rate as $\delta_{r}$, demand as $D_{r}$ and the deterioration rate as $d_{1}$, at time t, the following is the inventory level.

$$
\begin{aligned}
& \frac{\mathrm{d} Q_{r 1}(t)}{\mathrm{d} t}+d_{1} Q_{r 1}(t)=\delta_{r}-D_{r}, t_{m 2} \leq t \leq t_{r 1} \\
& \frac{\mathrm{d} Q_{r 2}(t)}{\mathrm{d} t}+d_{1} Q_{r 2}(t)=-D_{r}, t_{r 1} \leq t \leq T_{t} .
\end{aligned}
$$

From equation (4.37), one can get

$$
\begin{aligned}
Q_{r 1}(t) e^{d_{1} t} & =\int\left(\delta_{r}-D_{r}\right) e^{d_{1} t} \mathrm{~d} t+C_{r 1} \\
Q_{r 1}(t) \cdot e^{d_{1} t} & =\frac{\left(\delta_{r}-D_{r}\right)}{d_{1}} e^{d_{1} t}+C_{r 1} \\
\text { At } t & =t_{m 2}, Q_{r 1}(t)=0 \\
Q_{r 1}(t) & =\frac{\left(\delta_{r}-D_{r}\right)}{d_{1}}\left\{1-e^{\left(t_{m 2}-t\right) d_{1}}\right\} .
\end{aligned}
$$

From equation (4.38), we get

$$
Q_{r 2}(t) \cdot e^{d_{1} t}=\left[-\frac{D_{r}}{d_{1}} e^{d_{1} t}\right]+C_{r 2}
$$




$$
\begin{aligned}
\text { At } t & =T_{t}, Q_{r 2}(t)=0 \\
Q_{r 2}(t) & =\left(\frac{D_{r}}{d_{1}}\right)\left(e^{\left(T_{t}-t\right) d_{1}}-1\right) .
\end{aligned}
$$

Several costs of remanufacturing cycle for the supplier are listed below.

Setup cost $C r_{\mathrm{SC}}=C_{\mathrm{SCR}}$.

Production cost $C r_{\mathrm{PDC}}=C_{\mathrm{PDCR}} \int_{t_{m 2}}^{t_{r 1}} \delta_{r} \mathrm{~d} t$

$$
=C_{\mathrm{PDCR}} \delta_{r}\left(t_{r 1}-t_{m 2}\right) \text {. }
$$

Procurement cost $C r_{\mathrm{PC}}=C_{\mathrm{PCR}} \int_{t_{m 2}}^{t_{r 1}} Q_{r 1}(t) \mathrm{d} t$

$$
=C_{\mathrm{PCR}} \frac{\left(\delta_{r}-D_{r}\right)}{d_{1}}\left\{\left(t_{r 1}-t_{m 2}\right)+\frac{1}{d_{1}}\left(e^{\left(t_{m 2}-t_{r 1}\right) d_{1}}-1\right)\right\} .
$$

Deterioration cost $C r_{\mathrm{DC}}=\left(C_{\mathrm{DCR}}+C_{\mathrm{DCR} 1}\right)\left[\int_{t_{m 2}}^{t_{r 2}} d_{1} Q_{r 1}(t) \mathrm{d} t+\int_{t_{r 2}}^{T_{t}} d_{1} Q_{r 2}(t) \mathrm{d} t\right]$

$$
=\left(C_{\mathrm{DCR}}+C_{\mathrm{DCR} 1}\right)\left[\begin{array}{l}
\delta_{r}\left(t_{r 2}-t_{m 2}\right)-D_{r}\left(T_{t}-t_{m 2}\right)+\frac{\delta_{r}}{d_{1}}\left(e^{\left(t_{m 2}-t_{r 2}\right) d_{1}}-1\right) \\
-\frac{D_{r}}{d_{1}}\left(e^{\left(t_{m 2}-t_{r 2}\right) d_{1}}-e^{\left(T_{t}-t_{r 2}\right) d_{1}}\right)
\end{array}\right] .
$$

Holding cost $C r_{\mathrm{HC}}=\left(C_{\mathrm{HCR}}+C_{\mathrm{HCR} 1}\right)\left[\int_{t_{m 2}}^{t_{r 1}} Q_{r 1}(t) \mathrm{d} t+\int_{t_{r 1}}^{T_{t}} Q_{r 2}(t) \mathrm{d} t\right]$

$$
=\left(C_{\mathrm{HCR}}+C_{\mathrm{HCR} 1}\right)\left(\frac{1}{d_{1}}\right)\left[\begin{array}{l}
\delta_{r}\left(t_{r 1}-t_{m 2}\right)+D_{r}\left(t_{m 2}-T_{t}\right)+\frac{\delta_{r}}{d_{1}}\left(e^{\left(t_{m 2}-t_{r 1}\right) d_{1}}-1\right) \\
+\frac{D_{r}}{d_{1}}\left(e^{\left(T_{t}-t_{r 1}\right) d_{1}}-e^{\left(t_{m 2}-t_{r 1}\right) d_{1}}\right)
\end{array}\right] .
$$

The total average cost of the supplier for remanufacturing cycle is calculated as follows:

$$
(\mathrm{TC} r)_{R}=\frac{1}{T_{t}-t_{m 2}}\left(C r_{\mathrm{SC}}+C r_{\mathrm{PDC}}+C r_{\mathrm{PC}}+C r_{\mathrm{DC}}+C r_{\mathrm{HC}}\right) .
$$

\subsection{Retailer's model during remanufacturing cycle}

By assuming $d_{2}$ as the rate of deterioration and demand is a linear function, the level of inventory at time $t$ is given as

$$
\begin{aligned}
\frac{\mathrm{d} Q_{r}(t)}{\mathrm{d} t}+d_{2} Q_{r}(t) & =-(p+q t), 0 \leq t \leq t_{r} \\
\text { At } t & =t_{r}, Q_{r}(t)=0 \\
Q_{r}(t) & =\left(\frac{1}{d_{2}}\right)\left[\left(\frac{q}{d_{2}}-p-q t\right)+e^{\left(t_{r}-t\right) d_{2}}\left(p+q t_{r}-\frac{q}{d_{2}}\right)\right] .
\end{aligned}
$$

The following are the different retailer costs for remanufacturing cycle.

Ordering cost $\mathrm{CR}_{\mathrm{OCR}}=C_{\mathrm{OCRR}}$.

Purchasing cost $\mathrm{CR}_{\mathrm{PCR}}=C_{\mathrm{PCRR}} Q_{r}(t=0)$

$$
=C_{\mathrm{PCRR}}\left(\frac{1}{d_{2}}\right)\left[\left(\frac{q}{d_{2}}-p\right)+e^{t_{r} d_{2}}\left(p+q t_{r}-\frac{q}{d_{2}}\right)\right] .
$$

Deterioration cost $\mathrm{CR}_{\mathrm{DCR}}=\left(C_{\mathrm{DCRR}}+C_{\mathrm{DCRR} 1}\right) \int_{0}^{t_{r}} d_{2} Q_{r}(t) \mathrm{d} t$ 


$$
=\left(C_{\mathrm{DCRR}}+C_{\mathrm{DCRR} 1}\right)\left[\left(\frac{q t_{r}}{d_{2}}-p t_{r}-\frac{q t_{r}^{2}}{2}\right)+\frac{1}{d_{2}}\left(p+q t_{r}-\frac{q}{d_{2}}\right)\left(e^{t_{r} d_{2}}-1\right)\right] .
$$

Holding cost $\mathrm{CR}_{\mathrm{HCR}}=\left(C_{\mathrm{HCRR}}+C_{\mathrm{HCRR} 1}\right) \int_{0}^{t_{r}} Q_{r}(t) \mathrm{d} t$

$$
=\left(C_{\mathrm{HCRR}}+C_{\mathrm{HCRR} 1}\right)\left(\frac{1}{d_{2}}\right)\left[\left(\frac{q t_{r}}{d_{2}}-p t_{r}-\frac{q t_{r}^{2}}{2}\right)+\frac{1}{d_{2}}\left(p+q t_{r}-\frac{q}{d_{2}}\right)\left(e^{t_{r} d_{2}}-1\right)\right] .
$$

The total average cost for a retailer during the remanufacturing cycle is calculated as follows:

$$
(\mathrm{TCR} r)_{R}=\frac{1}{t_{r}}\left(\mathrm{CR}_{\mathrm{OCR}}+\mathrm{CR}_{\mathrm{PCR}}+\mathrm{CR}_{\mathrm{DCR}}+\mathrm{CR}_{\mathrm{HCR}}\right) .
$$

This study assumes that the total cycles of retailer for the remanufacturing cycle is $C_{2}$. Cycle time for the retailer is $t_{r}$. So, $t_{r}$ is written as:

$$
t_{r}=\frac{T_{t}-t_{m 2}}{C_{2}}
$$

As a consequence, the overall average cost of the remanufacturing cycle is calculated as follows:

$$
\begin{aligned}
& \text { Total cost }\left[\mathrm{TC}_{R}\left(t_{r 1}, T_{t}\right)\right]= \\
& \qquad \begin{aligned}
\mathrm{TC}_{R}\left(t_{r 1}, T_{t}\right)= & \frac{1}{T_{t}-t_{m 2}}\left(C r_{\mathrm{SC}}+C r_{\mathrm{PDC}}+C r_{\mathrm{PC}}+C r_{\mathrm{DC}}+C r_{\mathrm{HC}}\right) \\
& +\frac{C_{2}}{T_{t}-t_{m 2}}\left(\mathrm{CR}_{\mathrm{OCR}}+\mathrm{CR}_{\mathrm{PCR}}+\mathrm{CR}_{\mathrm{DCR}}+\mathrm{CR}_{\mathrm{HCR}}\right) .
\end{aligned}
\end{aligned}
$$

\section{Solution methodology}

The prime goal of this work is to reduce the overall cost of manufacturing and remanufacturing cycles in the provided equations (A.1) and (A.2). (See Appendix A)

The manufacturing part is processed in Mathematica-9 software under $0<t_{m 1}<t_{m 2}$. Put $t_{m}=\frac{t_{m 2}}{C_{1}}$ and other parameters. Therefore, find the values $t_{m 1}^{*}, t_{m 2}^{*}$, and minimize total cost.

The remanufacturing part is processed in Mathematica-9 software under $0<t_{r 1}<T_{t}$ and satisfied Hessian matrix.

This research gets the following result from equation (A.2).

$$
\frac{\partial \mathrm{TC}_{R}}{\partial t_{r 1}}=0 \quad \text { and } \quad \frac{\partial \mathrm{TC}_{R}}{\partial \mathrm{T}_{t}}=0 .
$$

It is difficult to identify the optimal values of $t_{r 1}^{*}$ and $\mathrm{T}_{t}^{*}$; hence, the Mathematica software is utilized to do it.

The Hessian matrix, $H_{\mathrm{RC}}=\operatorname{det}\left(\begin{array}{ll}\frac{\partial^{2} \mathrm{TC}_{R}}{\partial t_{r 1}^{2}} & \frac{\partial^{2} \mathrm{TC}_{R}}{\partial t_{r 1} \partial \mathrm{T}_{t}} \\ \frac{\partial^{2} \mathrm{TC}_{R}}{\partial t_{r 1} \partial \mathrm{T}_{t}} & \frac{\partial^{2} \mathrm{TC}_{R}}{\partial \mathrm{T}_{t}^{2}}\end{array}\right)>0$ and $\frac{\partial^{2} \mathrm{TC}_{R}}{\partial t_{r 1}^{2}}>0, \frac{\partial^{2} \mathrm{TC}_{R}}{\partial \mathrm{T}_{t}^{2}}>0$ satisfies the minimization function's sufficient condition.

The total minimum-cost function for remanufacturing is then calculated using equation (A.2).

\section{Algorithm}

(1) First, the total cost is determined as $\mathrm{TC}_{R}\left(t_{m}, t_{R}\right)$ using the value of the total cost function at $t_{r}=\frac{T_{t}-t_{r 1}}{C_{2}}$.

(2) The values of $t_{r 1}^{*}$ and $\mathrm{T}_{t}^{*}$ are then determined by considering the required criteria $\frac{\partial \mathrm{TC}_{R}}{\partial t_{r 1}}=0$ and $\frac{\partial \mathrm{TC}_{R}}{\partial \mathrm{T}_{t}}=0$. 
TABLE 2. Total cost during manufacturing and remanufacturing.

\begin{tabular}{llllll}
\hline & \multicolumn{2}{c}{ During manufacturing } & \multicolumn{3}{c}{ During remanufacturing } \\
\hline$t_{m 1}$ & $t_{m 2}$ & Total cost (\$/time unit) & $t_{r 1}$ & $T_{t}$ & Total cost (\$/time unit) \\
\hline 0.41 & 0.46 & 20562.4 & 0.98 & 1.14 & 3261.21 \\
\hline
\end{tabular}

(3) The Hessian matrix is satisfied by the sufficient condition as follows:

$$
H_{\mathrm{RC}}=\operatorname{det}\left(\begin{array}{cc}
\frac{\partial^{2} \mathrm{TC}_{R}}{\partial t_{r 1}^{2}} & \frac{\partial^{2} \mathrm{TC}_{R}}{\partial t_{r 1} \partial \mathrm{T}_{t}} \\
\frac{\partial^{2} \mathrm{TC}_{R}}{\partial t_{r 1} \partial \mathrm{T}_{t}} & \frac{\partial^{2} \mathrm{TC}_{R}}{\partial \mathrm{T}_{t}^{2}}
\end{array}\right)>0 \quad \text { and } \quad \frac{\partial^{2} \mathrm{TC}_{R}}{\partial t_{r 1}^{2}}>0, \frac{\partial^{2} \mathrm{TC}_{R}}{\partial \mathrm{T}_{t}^{2}}>0 .
$$

(4) This research then determines the values of $t_{m 1}, t_{m 2}, t_{r 1}$, and $T_{t}$.

(5) Lastly, this research determines manufacturing and remanufacturing's minimum value.

\section{NumERICAL EXAMPLE}

This section determines the optimal time and minimum total cost by utilizing Mathematica-9.0 as well as necessary input parameter values $[25,29]$.

Example 1. The manufacturing cycle take value as $C_{\mathrm{SCM}}=92 \$ /$ setup, $C_{\mathrm{ACM}}=81 \$ /$ advertisement, $a_{0}=24$, $a_{1}=11, a_{2}=0.25, C_{\mathrm{PDCM}}=74 \$ /$ unit, $C_{\mathrm{PCM}}=10 \$ /$ unit, $\alpha=77$ (units/unit time), $d_{1}=0.9, D_{0}=34$ units, $\beta=0.5$ (units/unit time), $\lambda=0.27, C_{\mathrm{DCM}}=15 \$ /$ unit, $C_{\mathrm{DCM} 1}=3 \$ /$ unit, $C_{\mathrm{HCM}}=77 \$ /$ unit $/$ unit time, $C_{\mathrm{HCM} 1}=3 \$ /$ unit/unit time, $C_{1}=2, C_{\mathrm{OCRM}}=89 \$ /$ order, $C_{\mathrm{PCRM}}=66.5 \$ /$ unit, $p=55$ (units/unit time), $q=0.1$ (units/unit time), $d_{2}=0.3, C_{\mathrm{DCRM}}=44 \$ /$ unit, $C_{\mathrm{DCRM} 1}=6, C_{\mathrm{HCRM}}=45 \$ /$ unit $/$ unit time, $C_{\mathrm{HCRM} 1}=3 \$ /$ unit/unit time. The best possible value of total cost is $20562.4, t_{m 1}$ is 0.41 and $t_{m 2}$ is 0.46 . $t_{m}$ can be found out from equation (4.33) and $t_{m}=\frac{t_{m 2}}{C_{1}}$.

Example 2. The remanufacturing cycle take value as $C_{\mathrm{SCR}}=198 \$ / \mathrm{setup}, C_{\mathrm{PDCR}}=175 \$ /$ unit, $C_{\mathrm{PCR}}=10 \$ /$ unit, $\alpha=97$ (units/unit time), $d_{1}=0.9, \delta_{r}=89$ (units/unit time), $\beta=0.5$ (units/unit time), $\lambda=0.27, C_{\mathrm{DCR}}=36 \$ /$ unit, $C_{\mathrm{DCR} 1}=4 \$ /$ unit, $C_{\mathrm{HCR}}=60 \$ /$ unit/unit time, $C_{\mathrm{HCR} 1}=5 \$ / \mathrm{unit} /$ unit time, $C_{2}=10$ cycle, $C_{\mathrm{OCRR}}=84 \$ /$ order,$C_{\mathrm{PCRR}}=195 \$ /$ unit, $p=55$ (units/unit time), $q=0.1$ (units $/$ unit time), $d_{2}=0.3, C_{\mathrm{DCRR}}=40 \$ /$ unit, $C_{\mathrm{DCRR} 1}=5 \$ /$ unit, $C_{\mathrm{HCRR}}=60 \$ /$ unit $/$ unit time, $C_{\mathrm{HCRR} 1}=4 \$ /$ unit $/$ unit time, $\eta_{r}=0.92, \mu_{r}=0.69, D_{r}=83, D_{0}=34, I_{c}=40, t_{m 2}=0.46$ unit time. Total cost has the best possible value of $3261.21, t_{r 1}$ is 0.98 , and $T_{t}$ is 1.14. Equation (74) and $t_{r}=\frac{T_{t}-t_{r 1}}{C_{2}}$ can be used to calculate $t_{r}$.

This study validates numerically through the Hessian matrix.

It satisfies condition $\operatorname{det}\left(\begin{array}{cc}\frac{\partial^{2} \mathrm{TC}_{R}}{\partial t_{r 1}^{2}} & \frac{\partial^{2} \mathrm{TC}_{R}}{\partial t_{r 1} \partial \mathrm{T}_{t}} \\ \frac{\partial^{2} \mathrm{TC}_{R}}{\partial t_{r 1} \partial \mathrm{T}_{t}} & \frac{\partial^{2} \mathrm{TC}_{R}}{\partial \mathrm{T}_{t}^{2}}\end{array}\right)>1.3 \times 10^{8}>$ and $\frac{\partial^{2} \mathrm{TC}_{2}}{\partial t_{r 1}^{2}}>7247.41>0, \frac{\partial^{2} \mathrm{TC}_{2}}{\partial T_{t}^{2}}>22358.8>0$ $\& \frac{\partial^{2} \mathrm{TC}_{2}}{\partial t_{r 1} \partial T_{t}}=-5410.03$ for the optimal value $t_{r 1}^{*}, T_{t}^{*}$.

\section{SEnsitivity ANALYSis}

This component of the research looks at the consequences of changing organization parameters in order to conduct a sensitivity of the planned model by regard to a few parameters. 


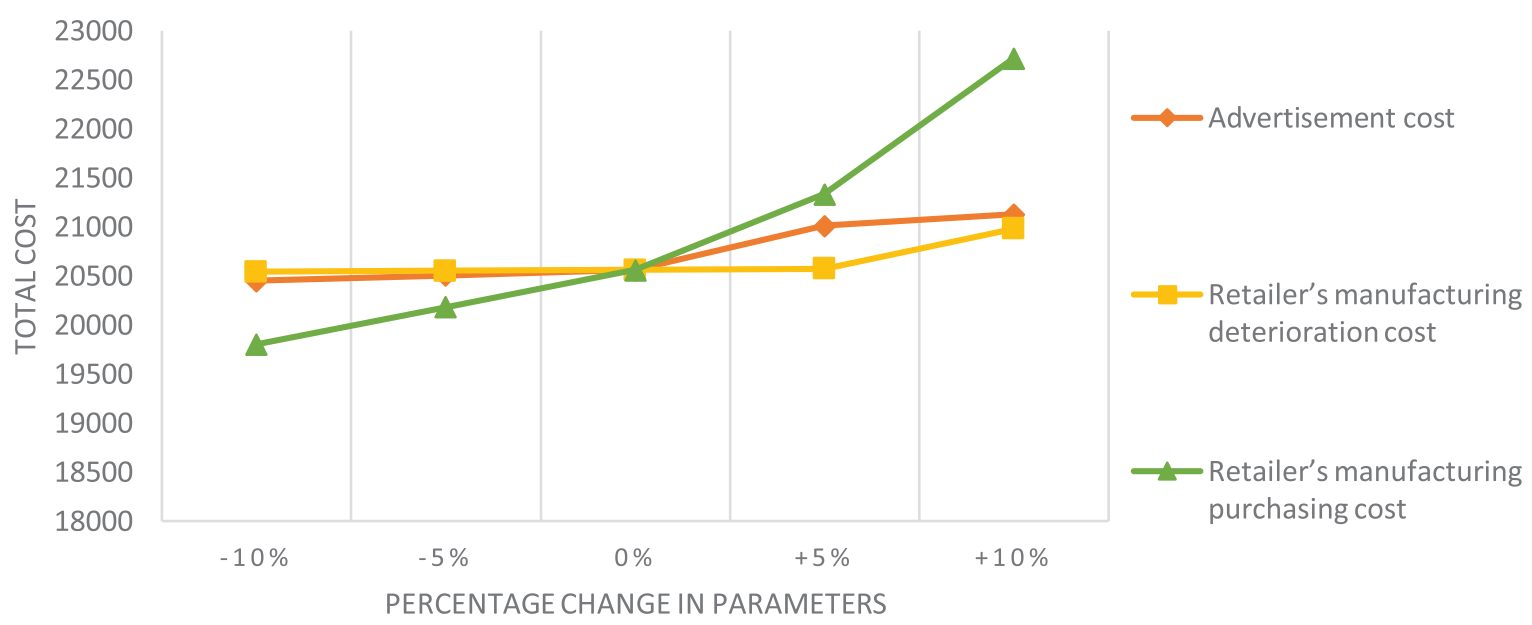

FIgURE 3. Flow of supplier advertisement cost, retailer's deterioration and purchasing cost during manufacturing cycle.

TABLE 3. Total cost change with respect to advertisement cost.

\begin{tabular}{lllll}
\hline & $\begin{array}{l}\text { Percentage } \\
\text { change }\end{array}$ & $t_{m 1}$ & $t_{m 2}$ & Total cost (\$/time unit) \\
\hline & $-10 \%$ & 0.41 & 0.46 & 20451.8 \\
& $-5 \%$ & 0.41 & 0.46 & 20507.1 \\
$C_{\mathrm{ACM}}(81(\$ /$ advertisement $))$ & $0 \%$ & 0.41 & 0.46 & 20562.4 \\
& $+5 \%$ & 0.34 & 0.37 & 21012.6 \\
& $+10 \%$ & 0.34 & 0.37 & 21129.3 \\
\hline
\end{tabular}

TABLE 4. Total cost change with respect to retailer's manufacturing deterioration cost.

\begin{tabular}{lllll}
\hline & $\begin{array}{l}\text { Percentage } \\
\text { change }\end{array}$ & $t_{m 1}$ & $t_{m 2}$ & Total cost (\$/time unit) \\
\hline & $-10 \%$ & 0.41 & 0.46 & 20543.4 \\
& $-5 \%$ & 0.41 & 0.46 & 20552.9 \\
$C_{\text {DCRM }}(44$ (\$/unit) $)$ & $0 \%$ & 0.41 & 0.46 & 20562.4 \\
& $+5 \%$ & 0.44 & 0.46 & 20571.9 \\
& $+10 \%$ & 0.34 & 0.38 & 20980.4 \\
\hline
\end{tabular}

\section{Observations and managerial insights}

Results of manufacturing cycle are shown in Figure 3 and Tables 3-5, whereas the remanufacturing cycle's results are shown in Table 6, Figures 4-6. The following are the results of this study.

(i) Figure 4 shows that adjusting the cost parameters $C_{\mathrm{SCR}}$ and $C_{\mathrm{PCR}}$ by a percentage reduces the overall cost of remanufacturing cycle. As a result, as the deterioration parameter $C_{\mathrm{DCR}}$ is modified by a percentage, the overall cost is increased.

(ii) Table 3 demonstrates that a very tiny drop follows a change in advertising cost in manufacturing time $t_{m 1}$ and $t_{m 2}$, while the total cost is increased. 
TABLE 5. Total cost change with respect to retailer's manufacturing purchasing cost.

\begin{tabular}{lllll}
\hline & $\begin{array}{l}\text { Percentage } \\
\text { change }\end{array}$ & $t_{m 1}$ & $t_{m 2}$ & Total cost (\$/time unit) \\
\hline & $-10 \%$ & 0.41 & 0.46 & 19802.7 \\
& $-5 \%$ & 0.41 & 0.46 & 20182 \\
$C_{\text {PCRM }}(66.5(\$ /$ unit $))$ & $0 \%$ & 0.41 & 0.46 & 20562.4 \\
& $+5 \%$ & 0.34 & 0.37 & 21332.7 \\
& $+10 \%$ & 0.35 & 0.40 & 22721.3 \\
\hline
\end{tabular}

TABLE 6. Total cost change with respect to change in various parameters.

\begin{tabular}{llllll}
\hline & \multicolumn{5}{c}{ Total cost $(\$ /$ time unit) } \\
\cline { 2 - 6 } Parameters & $-10 \%$ & $-5 \%$ & $0 \%$ & $+5 \%$ & $+10 \%$ \\
\hline$C_{\mathrm{SCR}}$ & 3298.36 & 3279.48 & 3261.21 & 3243.55 & 3226.52 \\
$C_{\mathrm{PCR}}$ & 3378.11 & 3319.5 & 3261.21 & 3203.25 & 3145.62 \\
$C_{\mathrm{DCR}}$ & 3246.98 & 3253007 & 3261.21 & 3271.31 & 3283.29 \\
$C_{\mathrm{HCR}}$ & 3242.25 & 3248.78 & 3261.21 & 3279.09 & 3302.00 \\
$C_{\mathrm{PCRR}}$ & 2924.37 & 3092.71 & 3261.21 & 3430.27 & 3600.26 \\
$p$ & 2900.43 & 3090.63 & 3261.21 & 3432.59 & 3605.16 \\
$q$ & 3261.18 & 3261.19 & 3261.21 & 3261.22 & 3261.24 \\
$C_{\text {DCRR }}$ & 3262.26 & 3261.74 & 3261.21 & 3260.68 & 3260.16 \\
$C_{\mathrm{HCRR}}$ & 3255.96 & 3258.58 & 3261.21 & 3263.84 & 3265.60 \\
\hline
\end{tabular}

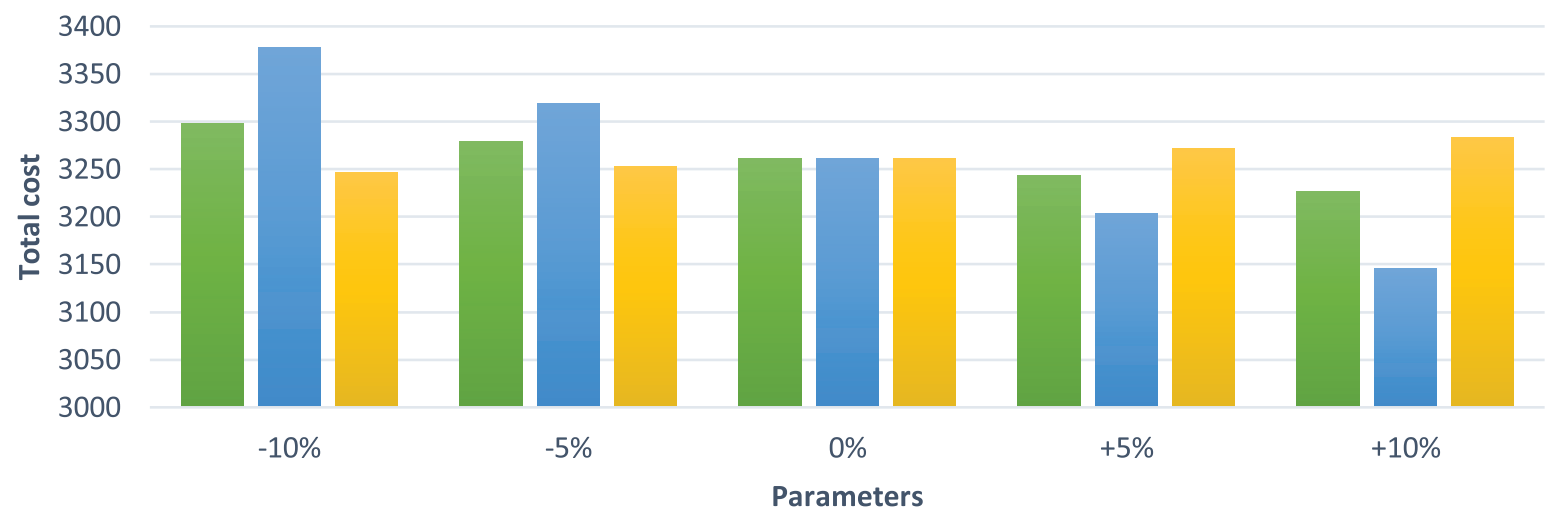

Remaufacturing's set up cost $\quad$ Remaufacturing's procurement cost $\square$ Remaufacturing's deterioration cost

Figure 4. Flow of remanufacturing's setup, procurement and deterioration cost.

(iii) As shown in Figure 5, the overall cost of remanufacturing cycle increases due to percentage increases $(-10$, $-5,0,+5$, and $+10 \%)$ in the output parameters $C_{\mathrm{HCR}}, C_{\mathrm{PCRR}}$ and $p$.

(iv) When the manufacturing deterioration cost of a retailer $C_{\mathrm{DCRM}}$ is changed, the manufacturing time $t_{m 1}$ and $t_{m 2}$ remain unchanged or decrease, resulting in a total cost rise as shown in Table 4. 


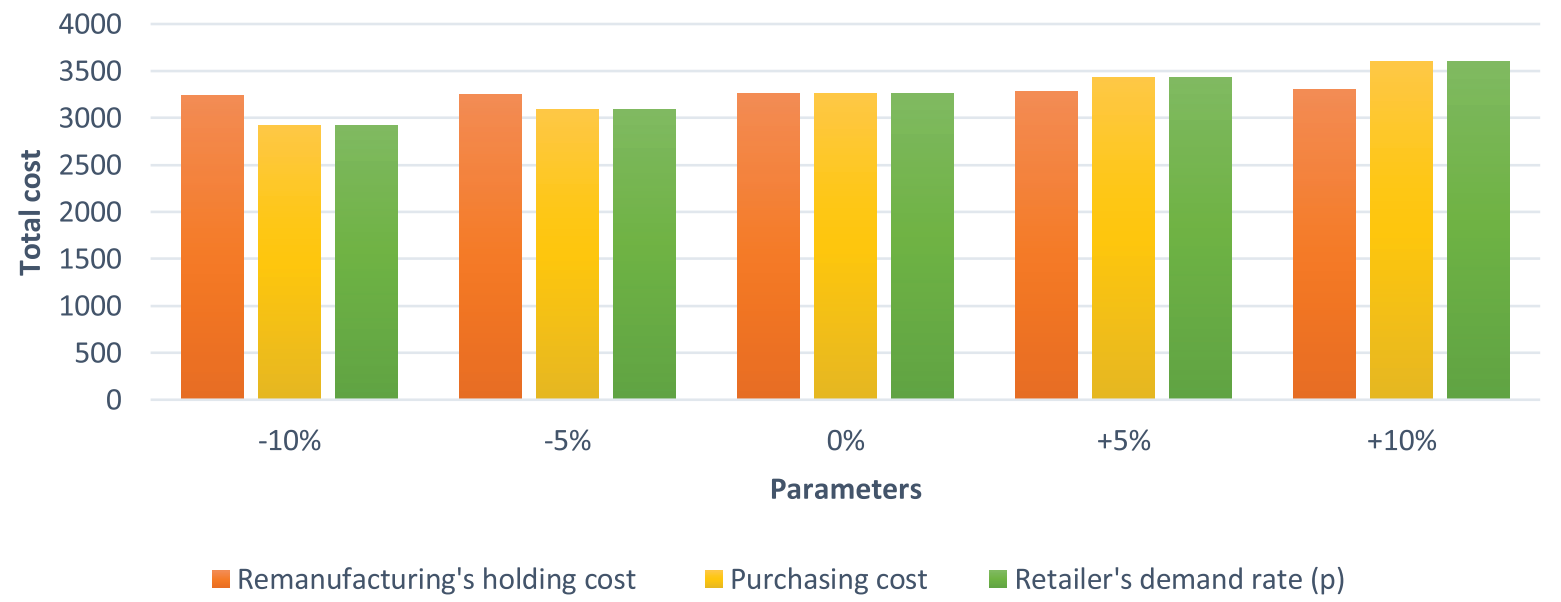

FigURE 5. Flow of remanufacturing's holding cost, retailer's remanufacturing purchasing cost and demand rate $(p)$.

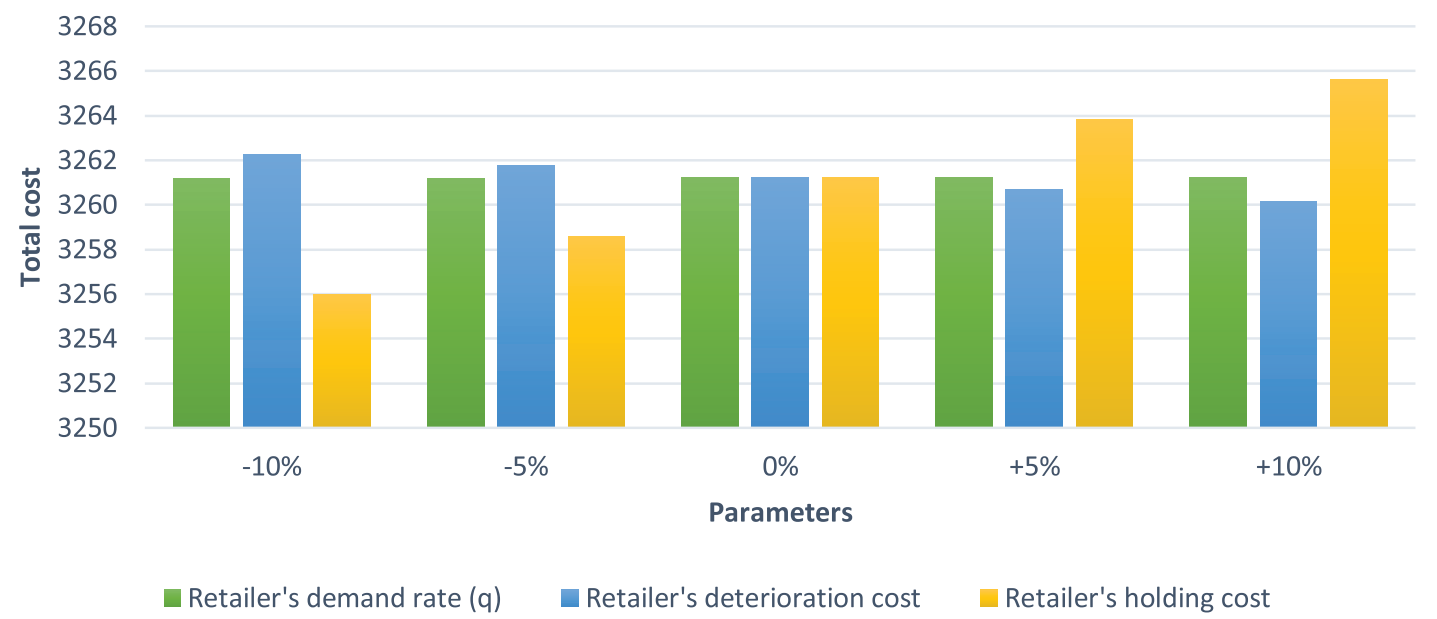

FiguRE 6. Flow of retailer's remanufacturing demand rate $(q)$, deterioration and holding cost.

(v) The overall cost of remanufacturing is increased when the parameters $q$ and $C_{\mathrm{HCRR}}$ are modified by a percentage in Figure 6. The overall cost of remanufacturing cycle is reduced as the deterioration cost parameter $C_{\mathrm{DCRR}}$ is moved from negative to positive by a percentage change.

(vi) Figure 3 indicates that the manufacturing cycle overall cost increases when the parameter $C_{\mathrm{PCRM}}$ is adjusted by a percentage.

\section{Conclusions}

The advertisement has a positive effect on customer demand. This method considers several facets of the green supply chain, such as waste collection, reverse logistics, and remanufacturing. Items that have been remanufactured are thought to be as excellent as new products. The manufacturing and remanufacturing models are solved individually because remanufacturing has its own set of costs, such as new infrastructure and labor, 
and modeling involves considering the collection cycle. A supply chain inventory framework is designed in this work to pick the optimum total cost. It allows analysis remanufacturing separately and assess its impact.

The finding confirms that as advertising costs rise, the total cost rises. There is a significant increase in the market's worth. As a result, the overall cost throughout the manufacturing cycle is higher than the overall cost during the remanufacturing cycle, as there is an item of expenditure on the advertisement cost during the manufacturing cycle. Furthermore, the holding and deterioration costs are spent on carbon emissions, which is excellent for the environment. Some parameters show fluctuations. Thus the necessary parameters are set to a certain value to evaluate the model's feasibility. This research can be useful in the smart products and garment industries in the real world.

The sensitivity analysis and case study yield substantial managerial insights. Advertisements have a significant beneficial effect on industries by providing immediate information to the customer. As a result, the demand for industrial items is growing rapidly. This proposed study, which primarily consists of research on different reverse logistics services and techniques in the industries, has been used for both manufacturing and remanufacturing, but it does have some limitations. This study has not taken the same decision variables for manufacturing and remanufacturing cycles. The presented model can be modified in numerous ways for further research, including (1) allowing for trade-credit in the vendor-buyer integration (Vandana et al. [52]) and (2) extending this model for stock-dependent demand, shortage and backlog, inflation, and selling price. (3) This model can be modified to include a fuzzy environment (Mahapatra et al. [46]). (4) This study can be extended to achieve environmental sustainability, such as Sarkar et al. [36], Bhuniya et al.[4] and Kumar et al. [22].

\section{Appendix A.}

$$
\begin{aligned}
& \mathrm{TC}_{M}\left(t_{m 1}, t_{m 2},\right)=\frac{1}{t_{m 2}}\left\{C_{\mathrm{SCM}}+C_{\mathrm{ACM}}\left(\frac{\left(-1+e^{-a_{2} t_{m 2}}\right) a_{1}}{a_{2}}+a_{0} t_{m 2}\right)+C_{\mathrm{PDCM}}\left(\frac{\beta t_{m 1}^{2}}{2}+\alpha \mathrm{t}_{m 1}\right)\right. \\
& +C_{\mathrm{PCM}}\left(\frac{a_{1}}{\left(\lambda-a_{2}\right)\left(a_{2}-d_{1}\right)}\left(\frac{-1+e^{-a_{2} t_{m 1}}}{a_{2}}+\frac{1-e^{-d_{1} t_{m 1}}}{d_{1}}\right)+\left(\frac{-1+e^{-\lambda t_{m 1}}}{\lambda}+\frac{1-e^{-d_{1} t_{m 1}}}{d_{1}}\right)\right. \\
& \left.\times\left(-\frac{a_{0}}{\lambda}+\frac{a_{1}}{\lambda-a_{2}}+D_{0}\right) \frac{1}{\left(-\lambda+d_{1}\right)}+\frac{\beta t_{m 1}^{2}}{2 d_{1}}+\left(\alpha-\frac{a_{0}}{\lambda}-\frac{\beta}{d_{1}}\right)\left(\frac{-1+e^{-d_{1} t_{m 1}}}{d_{1}}+t_{m 1}\right) \frac{1}{d_{1}}\right) \\
& +\left(C_{\mathrm{DCM}}+C_{\mathrm{DCM} 1}\right) d_{1}\left(-\frac{e^{-\lambda t_{m 1}}-e^{-\lambda t_{m 2}}}{\lambda}-\frac{\left(e^{-a_{2} t_{m 1}}-e^{-a_{2} t_{m 2}}\right) a_{1}}{\left(\lambda-a_{2}\right) a_{2}\left(a_{2}-d_{1}\right)}\right. \\
& +\frac{a_{1}\left(\frac{-1+e^{-a_{2} t_{m 1}}}{a_{2}}+\frac{1-e^{-d_{1} t_{m 1}}}{d_{1}}\right)}{\left(\lambda-a_{2}\right)\left(a_{2}-d_{1}\right)}+\frac{\left(\frac{-1+e^{-\lambda t_{m 1}}}{\lambda}+\frac{1-e^{-d_{1} t_{m 1}}}{d_{1}}\right)\left(-\frac{a_{0}}{\lambda}+\frac{a_{1}}{\lambda-a_{2}}+D_{0}\right)}{-\lambda+d_{1}} \\
& +\frac{a_{0} t_{m 1}}{\lambda\left(-\lambda+d_{1}\right)}+\frac{D_{0} t_{m 1}}{\lambda-d_{1}}+\frac{\beta t_{m 1}^{2}}{2 d_{1}}+\frac{\left(\alpha-\frac{a_{0}}{\lambda}-\frac{\beta}{d_{1}}\right)\left(\frac{-1+e^{-d_{1} t_{m 1}}}{d_{1}}+t_{m 1}\right)}{d_{1}}+\frac{a_{1}\left(t_{m 1}-t_{m 2}\right)}{\left(\lambda-a_{2}\right)\left(\lambda-d_{1}\right)} \\
& +\frac{e^{\left(-a_{2}+d_{1}\right) t_{m 2}} a_{1}\left(t_{m 1}-t_{m 2}\right)}{\left(-\lambda+a_{2}\right)\left(a_{2}-d_{1}\right)}+\frac{a_{0} t_{m 2}}{\lambda^{2}-\lambda d_{1}}+\frac{D_{0} t_{m 2}}{-\lambda+d_{1}}+e^{\left(-a_{2}+d_{1}\right) t_{m 2}}\left(-t_{m 1}+t_{m 2}\right) \\
& \left.+\frac{e^{-1+d_{1} t_{m 2}} a_{0}\left(-t_{m 1}+t_{m 2}\right)}{\lambda d_{1}}\right)+\left(C_{\mathrm{HCM}}+C_{\mathrm{HCM} 1}\right)\left(-\frac{e^{-\lambda t_{m 1}}-e^{-\lambda t_{m 2}}}{\lambda}-\frac{\left(e^{-a_{2} t_{m 1}}-e^{-a_{2} t_{m 2}}\right) a_{1}}{\left(\lambda-a_{2}\right) a_{2}\left(a_{2}-d_{1}\right)}\right. \\
& +\frac{a_{1}\left(\frac{-1+e^{-a_{2} t_{m 1}}}{a_{2}}+\frac{1-e^{-d_{1} t_{m 1}}}{d_{1}}\right)}{\left(\lambda-a_{2}\right)\left(a_{2}-d_{1}\right)}+\frac{\left(\frac{-1+e^{-\lambda t_{m 1}}}{\lambda}+\frac{1-e^{-d_{1} t_{m 1}}}{d_{1}}\right)\left(-\frac{a_{0}}{\lambda}+\frac{a_{1}}{\lambda-a_{2}}+D_{0}\right)}{-\lambda+d_{1}} \\
& +\frac{a_{0} t_{m 1}}{\lambda\left(-\lambda+d_{1}\right)}+\frac{D_{0} t_{m 1}}{\lambda-d_{1}}+\frac{\beta t_{m 1}^{2}}{2 d_{1}}+\frac{\left(\alpha-\frac{a_{0}}{\lambda}-\frac{\beta}{d_{1}}\right)\left(\frac{-1+e^{-d_{1} t_{m 1}}}{d_{1}}+t_{m 1}\right)}{d_{1}}+\frac{a_{1}\left(t_{m 1}-t_{m 2}\right)}{\left(\lambda-a_{2}\right)\left(\lambda-d_{1}\right)}
\end{aligned}
$$




$$
\begin{aligned}
& +\frac{e^{\left(-a_{2}+d_{1}\right) t_{m 2}} a_{1}\left(t_{m 1}-t_{m 2}\right)}{\left(-\lambda+a_{2}\right)\left(a_{2}-d_{1}\right)}+\frac{a_{0} t_{m 2}}{\lambda^{2}-\lambda d_{1}}+\frac{D_{0} t_{m 2}}{-\lambda+d_{1}}+e^{\left(-a_{2}+d_{1}\right) t_{m 2}}\left(-t_{m 1}+t_{m 2}\right) \\
& \left.\left.+\frac{e^{-1+d_{1} t_{m 2}} a_{0}\left(-t_{m 1}+t_{m 2}\right)}{\lambda d_{1}}\right)\right\}+\frac{C_{1}}{t_{m}}\left\{C_{\mathrm{OCRM}}+C_{\mathrm{PCRM}}\left(\frac{\left(1-e^{d_{2} t_{m}}\right) q}{d_{2}^{2}}+\frac{\left(-1+e^{d_{2} t_{m}}\right) p}{d_{2}}\right.\right. \\
& \left.+\frac{e^{d_{2} t_{m}} q t_{m}}{d_{2}}\right)+\frac{\left(C_{\mathrm{HCRM}}+C_{\mathrm{HCRM} 1}\right)}{d_{2}^{3}}\left(2\left(-1+e^{d_{2} t_{m}}\right)+d_{2}\left(p-e^{d_{2} t_{m}} p+t_{m}\left(-e^{d_{2} t_{m}} q\right.\right.\right. \\
& \left.\left.\left.+e^{d_{2} t_{m}}\left(-q+d_{2}\left(p+q t_{m}\right)\right)\right)\right)\right)+\frac{\left(C_{\mathrm{DCM} 1}+C_{\mathrm{DCRM}}\right)}{d_{2}^{2}}\left(2\left(-1+e^{d_{2} t_{m}}\right) q+d_{2}\left(p-e^{d_{2} t_{m}} p\right.\right. \\
& \left.\left.+t_{m}\left(-e^{d_{2} t_{m}} q+e^{d_{2} t_{m}}\left(-q+d_{2}\left(p+q t_{m}\right)\right)\right)\right)\right\} \text {. } \\
& \mathrm{TC}_{R}\left(t_{r 1}, T_{t}\right)=\frac{1}{\left(T_{t}-t_{m 2}\right)}\left\{C_{\mathrm{SCR}}+C_{\mathrm{PDCR}} \delta_{r}\left(t_{r 1}-t_{m 2}\right)+C_{\mathrm{PCR}}\left(\frac{-1+e^{d_{1}\left(t_{m 2}-t_{r 1}\right)}}{d_{1}}\right.\right. \\
& \left.+\frac{\left(-t_{m 2}+t_{r 1}\right)\left(-D_{r}+\delta_{r}\right)}{d_{1}}\right)+\left(C_{\mathrm{DCR}}+C_{\mathrm{DCR} 1}\right)\left(\frac{\left(-1+e^{d_{1}\left(-t_{r 1}+T_{t}\right)}\right) D_{r}}{d_{1}}+D_{r}\left(t_{r 1}-T_{t}\right)\right. \\
& \left.+\frac{\left(-1+e^{d_{1}\left(t_{m 2}-t_{r 1}\right)}\right)\left(-D_{r}+\delta_{r}\right)}{d_{1}}+\left(-t_{m 2}+t_{r 1}\right)\left(-D_{r}+\delta_{r}\right)\right)+\frac{\left(C_{\mathrm{HCR}}+C_{\mathrm{HCR} 1}\right)}{d_{1}} \\
& \times\left(\frac{\left(-1+e^{d_{1}\left(-t_{r 1}+T_{t}\right)}\right) D_{r}}{d_{1}}+D_{r}\left(t_{r 1}-T_{t}\right)+\frac{\left(-1+e^{d_{1}\left(t_{m 2}-t_{r 1}\right)}\right)\left(-D_{r}+\delta_{r}\right)}{d_{1}}\right. \\
& \left.\left.+\left(-t_{m 2}+t_{r 1}\right)\left(-D_{r}+\delta_{r}\right)\right)\right\}+\frac{C_{2}}{\left(T_{t}-t_{m 2}\right)}\left\{C_{\mathrm{OCRR}}+C_{\mathrm{PCRR}}\left(\frac{\left(1-e^{d_{2} t_{r}}\right) q}{d_{2}^{2}}+\frac{\left(-1+e^{d_{2} t_{r}}\right) p}{d_{2}}\right.\right. \\
& \left.+\frac{e^{d_{2} t_{r}} q t_{r}}{d_{2}}\right)-\frac{\left(C_{\mathrm{DCRR}}+C_{\mathrm{DCRR} 1}\right)}{d_{2}^{2}}\left(2\left(-1+e^{d_{2} t_{r}}\right) q+d_{2}\left(p-e^{d_{2} t_{r}} p+t_{r}\left(-e^{d_{2} t_{r}} q\right.\right.\right. \\
& \left.\left.\left.+e^{d_{2} t_{r}}\left(-q+d_{2}\left(p+q t_{r}\right)\right)\right)\right)\right)+\frac{\left(C_{\mathrm{HCRR}}+C_{\mathrm{HCRR} 1}\right)}{d_{2}^{3}}\left(2\left(-1+e^{d_{2} t_{r}}\right) q\right. \\
& \left.\left.+d_{2}\left(p-e^{d_{2} t_{r}} p+t_{r}\left(-e^{d_{2} t_{r}} q+e^{d_{2} t_{r}}\left(-q+d_{2}\left(p+q t_{r}\right)\right)\right)\right)\right)\right\} \text {. }
\end{aligned}
$$

Acknowledgements. The first author Subhash Kumar is working at Meerut College,Meerut as a research scholar and thankful to UGC, India, for the financial support. The work is supported by the National Research Foundation of Korea (NRF) grant, funded by the Korea Government (MSIT) (NRF-2020R1F1A1064460).

\section{REFERENCES}

[1] A.A. Alamri, Theory and methodology on the global optimal solution to a general reverse logistics inventory model for decorating items and time-varying rates. Comput Ind. Eng. 60 (2010) 236-247.

[2] A. Aminipour, Z. Bahroun and M. Hariga, Cyclic manufacturing and remanufacturing in a closed-loop supply chain. Sust. Prod. Consum. 25 (2021) 43-59.

[3] S. Bhuniya, S. Pareek and B. Sarkar, A supply chain model with service level constraints and strategies under uncertainty. Alex. Eng. J. 60 (2021) 6035-6052.

[4] S. Bhuniya, S. Pareek, B. Sarkar and B.K. Sett, A smart production process for the optimum energy consumption with maintenance policy under a supply chain management. Processes 9 (2021) 19.

[5] J. Chai, Z. Qian, F. Wang and J. Zhu, Process innovation for green product in a closed loop supply chain with remanufacturing. To appear in: Ann. Oper. Res. (2021) 1-25. DOI: 10.1007/s10479-020-03888-y. 
[6] C.C. Chang, C.J. Lu and C. Te, Multi-stage supply chain production-inventory model with collaborative preservation technology investment. To appear in: Sci. Iran. (2020). DOI: 10.24200/sci.2020.53357.3200.

[7] I.D. Cho, Analysis of optimal production and advertising policies. Int. J. Syst. Sci. 27 (1996) 1297-1305.

[8] K. Conrad, Price competition and product differentiation when consumers care for the environment. Environ. Resour. Eco. 31 (2005) 1-19.

[9] B.K. Dey, S. Bhuniya and B. Sarkar, Involvement of controllable lead time and variable demand for a smart manufacturing system under a supply chain management. Expert Syst. Appl. 184 (2021) 115464.

[10] B. Dey, S. Pareek, M. Tayyab and B. Sarkar, Autonomation policy to control work-in-process inventory in a smart production system. Int. J. Prod. Res. 59 (2021) 1258-1280.

[11] W.A. Donaldson, Inventory replenishment policy for a linear trend in demand: an analytical solution. Opers. Res. Q. 28 (1977) 663-670.

[12] A.M.A. El Saadany and M.Y. Jaber, Reproduction/remanufacturing inventory model with price and quality dependent return rate. Comput. Ind. Eng. 58 (2010) 352-362.

[13] A.M.A. El Saadany, M.Y. Jaber and M. Bonney, Environmental performance measures for supply chain. Manage. Res. Rev. 34 (2011) 1202-1221.

[14] L. Gennady, G.L. Brodetskiy, D.A. Gusev and I.G. Shidlovskii, Multi-criteria optimisation under the conditions of uncertainty in logistics and supply chain management. Int. J. Logist. Syst. Manage. 39 (2021) 207-227.

[15] S.K. Goyal and B.C. Giri, The production inventory problem of a product with time varying demand, production and deteriorates. Eur. J. Oper. Res. 145 (2003) 635-644.

[16] M.S. Habib, O. Asghar, A. Hussian, M. Imran, M.P. Mughal and B. Sarkar, A robust possibilistic programming approach toward animal fat-based biodiesel supply chain network design under uncertain environment. J. Clean. Prod. 278 (2021) 122403.

[17] S. Hazari, K. Maity, J.K. Dey and S. Kar, Advertisement policy and reliability dependent imperfect production inventory control problem in bi-fuzzy environment. Int. J. Oper. Res. 22 (2015) 342-365.

[18] K. Inderfurth, A. Janiak, M.Y. Kovalyov and F. Werner, Batching work and rework processes with limited deterioration of reworkable. Comput. Oper. Res. 33 (2006) 1595-1605.

[19] M. Karimi-Nasab, S. Dowlatshahi and H. Heidari, A multiobjective distribution-pricing model for multiperiod price sensitive demands. IEEE Trans. Eng. Manage. 60 (2013) 640-651.

[20] M.A.A. Khan, A.A. Shaikh, I. Konstantaras, A.K. Bhunia and L.E. Cárdenas-Barrón, Inventory models for perishable items with advanced payment, linearly time-dependent holding cost and demand dependent on advertisement and selling price. Int. J. Prod. Econ. 230 (2020) 107804.

[21] S. Kumar, A. Kumar and M. Jain, Learning effect on an optimal policy for mathematical inventory model for decaying items under preservation technology with the environment of COVID-19 pandemic. Malaya J. Mat. 8 (2020) 1694-1702.

[22] S. Kumar, B. Sarkar and A. Kumar, Fuzzy reverse logistics inventory model of smart items with two warehouses of a retailer considering carbon emissions. RAIRO-Oper. Res. 55 (2021) 2285-2307.

[23] Z. Liu, J. Chen, C. Diallo and U. Venkatadri, Pricing and production decisions in a dual-channel closed-loop supply chain with (re) manufacturing. Int. J. Prod. Econ. 232 (2021) 107935.

[24] W.Y. Lo, C.H. Tsai and R.K. Li, Exact solution of inventory replenishment policy for a linear trend in demand- two-equation model. Int. J. Prod. Econ. 76 (2002) 111-120.

[25] A.K. Manna, J.K. Dey and S.K. Mondal, Imperfect production inventory model with production rate dependent defective rate and advertisement dependent demand. Comput. Ind. Eng. 104 (2017) 9-22.

[26] S. Nahmias and H. Rivera, A deterministic model for a repairable item inventory system with finite repair rate. Int. J. Prod. Res. 17 (1979) 215-221.

[27] S. Rani, R. Ali and A. Agarwal, Green supply chain inventory model for that your rating items with valuable demand and the inflation. IJBAN 3 (2017) 50.

[28] W. Ahmed, M. Moazzam, B. Sarkar and S.U. Rehman, Synergic effect of reworking for imperfect quality items with the integration of multi-period delay-in-payment and partial backordering in global supply chains. Engineering 7 (2021) $260-271$.

[29] S. Rani, R. Ali and A. Agarwal, Fuzzy inventory model for deteriorating items in a green supply chain with carbon concerned demand. Opsearch 56 (2019) 91-122.

[30] K. Richter, The extended EOQ for repair and waste disposal model. Int. J. Prod. Econ. 45 (1996) $443-447$.

[31] K. Richter, The EOQ repair and waste disposal model with variable set up numbers. Eur. J. Oper. Res. 9 (1996) $313-324$.

[32] S. Saha, D. Chatterjee and B. Sarkar, The ramification of dynamic investment on the promotion and preservation technology for inventory management through a modified flower pollination algorithm. J. Retail. Consum. Serv. 58 (2021) 102326.

[33] B. Sarkar, M. Ullah and M. Sarkar, Environmental and economic sustainability through innovative green products by remanufacturing. J. Clean. Prod. 332 (2022) 129813.

[34] B. Sarkar, A. Debnath, A.S.F. Chiu and W. Ahmed, Circular economy-driven two-stage supply chain management for nullifying waste. J. Clean. Prod. 339 (2022) 130513.

[35] A.S.H. Kugele, W. Ahmed and B. Sarkar, Geometric programming solution of second degree difficulty for carbon ejection controlled reliable smart production system. To appear in: RAIRO Oper. Res. (2022). DOI: 10.1051/ro/2022028.

[36] B. Sarkar, M. Sarkar, B. Ganguly and L.E. Cárdenas-Barrón, Combined effects of carbon emission and production quality improvement for fixed lifetime products in a sustainable supply chain management. Int. J. Prod. Econ. 231 (2021) 107867. 
[37] N. Saxena, S.R. Singh and S.S. Sana, A green supply chain model of vendor and buyer for remanufacturing. RAIRO-Oper. Res. 51 (2017) 1133-1150.

[38] A. Garai and B. Sarkar, Economically independent reverse logistics of customer-centric closed-loop supply chain for herbal medicines and biofuel. J. Clean. Prod. 334 (2022) 129977.

[39] D.A. Schrady, A deterministic inventory model for repairable items. Nav. Res. Logist. Q. 14 (1967) $391-398$.

[40] M. Sebatjane and O. Adetunji, Optimal lot-sizing and shipment decisions in a three-echelon supply chain for growing items with inventory level-and expiration date-dependent demand. Appl. Math. Model. 90 (2021) 1204-1225.

[41] A. Sepehri, U. Mishra, M.L. Tseng and B. Sarkar, Joint pricing and inventory model for deteriorating items with maximum lifetime and controllable carbon emissions under permissible delay in payments. Mathematics 9 (2021) 470.

[42] E.A. Silver and H.C. Meal, A simple modification of the EOQ for the case of varying demand rate. Prod. Invent. Manage. 10 (1969) 52-65.

[43] E.A. Silver and R. Peterson, Decision systems for inventory management and production planning, 2nd edition. Wiley, New York (1985).

[44] S.R. Singh, D. Yadav, B. Sarkar and M. Sarkar, Impact of energy and carbon emission of a supply chain management with two-level trade-credit policy. Energies 14 (2021) 1569.

[45] M. Tayyab and B. Sarkar, An interactive fuzzy programming approach for a sustainable supplier selection under textile supply chain management. Comput. Ind. Eng. 155 (2021) 107164.

[46] A.S. Mahapatra, H.N. Soni, M.S. Mahapatra and B. Sarkar, A continuous review production-inventory system with a variable preparation time in a fuzzy random environment. Mathematics 9 (2021) 747.

[47] Y. Teng and B. Feng, Optimal channel structure for remanufacturing under cap-and-trade regulation. Processes 9 (2021) 370.

[48] D. Yadav, R. Kumari, N. Kumar and B. Sarkar, Reduction of waste and carbon emission through the selection of items with cross-price elasticity of demand to form a sustainable supply chain with preservation technology. J. Clean. Prod. 297 (2021) 126298.

[49] M. Ullah, I. Asghar, M. Zahid, M. Omair, A.A. Arjani and B. Sarkar, Ramification of remanufacturing in a sustainable three-echelon closed-loop supply chain management for returnable products. J. Clean. Prod. 290 (2021) 125609.

[50] R. UthayaKumar, K.V. Geetha and S.S. Sana, Economic ordering policy for non-instantaneous deteriorating items with price and advertisement dependent demand and permissible delay in payment under inflation. Math. Methods Appl. Sci. 44 (2021) $7697-7721$.

[51] G.A. Widyadana and H.M. Wee, An economic production quantity model for deteriorating items with multiple production setups and rework. Int. J. Prod. Econ. 138 (2012) 62-67.

[52] Vandana, S.R. Singh, D. Yadav, B. Sarkar and M. Sarkar, Impact of energy and carbon emission of a supply chain management with two-level trade-credit policy. Energies 14 (2021) 1569.

\section{Subscribe to Open (S2O) A fair and sustainable open access model}

This journal is currently published in open access under a Subscribe-to-Open model (S2O). S2O is a transformative model that aims to move subscription journals to open access. Open access is the free, immediate, online availability of research articles combined with the rights to use these articles fully in the digital environment. We are thankful to our subscribers and sponsors for making it possible to publish this journal in open access, free of charge for authors.

\section{Please help to maintain this journal in open access!}

Check that your library subscribes to the journal, or make a personal donation to the $\mathrm{S} 2 \mathrm{O}$ programme, by contacting subscribers@edpsciences.org

More information, including a list of sponsors and a financial transparency report, available at: https://www. edpsciences.org/en/maths-s2o-programme 